\title{
BURN CONTROL IN FUSION REACTORS VIA NONLINEAR STABILIZATION TECHNIQUES
}

\author{
EUGENIO SCHUSTER,* MIROSLAV KRSTIĆ, and GEORGE TYNAN \\ University of California, San Diego, Department of Mechanical and Aerospace Engineering \\ La Jolla, California 92093-0411
}

Received September 11, 2001

Accepted for Publication March 9, 2002

Control of plasma density and temperature magnitudes, as well as their profiles, are among the most fundamental problems in fusion reactors. Existing efforts on model-based control use control techniques for linear models. In this work, a zero-dimensional nonlinear model involving approximate conservation equations for the energy and the densities of the species was used to synthesize a nonlinear feedback controller for stabilizing the burn condition of a fusion reactor. The subignition case, where the modulation of auxiliary power and fueling rate are considered as control forces, and the ignition case, where the controlled injection of impurities is considered as an additional actuator, are treated separately.

The model addresses the issue of the lag due to the finite time for the fresh fuel to diffuse into the plasma center. In this way we make our control system independent of the fueling system and the reactor can be fed either by pellet injection or by puffing. This imposed lag is treated using nonlinear backstepping.
The nonlinear controller proposed guarantees a much larger region of attraction than the previous linear controllers. In addition, it is capable of rejecting perturbations in initial conditions leading to both thermal excursion and quenching, and its effectiveness does not depend on whether the operating point is an ignition or a subignition point.

The controller designed ensures setpoint regulation for the energy and plasma parameter $\beta$ with robustness against uncertainties in the confinement times for different species. Hence, the controller can increase or decrease $\beta$, modify the power, the temperature or the density, and go from a subignition to an ignition point and vice versa.

KEYWORDS: burn control, nonlinear control method, fusion reactor

\section{INTRODUCTION}

\section{I.A. Motivation}

In order to be commercially competitive, a fusion reactor needs to run long periods of time in a stable burning plasma mode at working points which are characterized by a high $Q$, where $Q$ is the ratio of fusion power to auxiliary power. Although operating points with these characteristics that are inherently stable exist for most confinement scalings, they are found in a region of high temperature and low density. Unfortunately, economical and technological constraints make these operating points unattractive and require the fusion reactor to operate in a zone of low temperature and high density

\footnotetext{
*E-mail: schuster@mae.ucsd.edu
}

where the thermonuclear reaction is inherently thermally unstable. Figure 1a shows that for low temperatures the rate of thermonuclear reaction for a D-T mixture increases as the plasma temperature rises. In this thermally unstable zone, a small increase of temperature leads to an increase of power which results in thermal excursion. Although the excursion reaches a stable uneconomical working point at a higher temperature, the plasma can be led to beta or density limit disruptions before reaching this point. On the other hand, a small decrease of temperature leads to a decrease of power and quenching. Even during a quenching, a disruptive instability can be reached, causing wall damage.

Some experimental conditions can be arranged in such a way that certain amount of passive stabilization of the thermonuclear reaction can be obtained. Nevertheless, this approach is often insufficient and even authors 


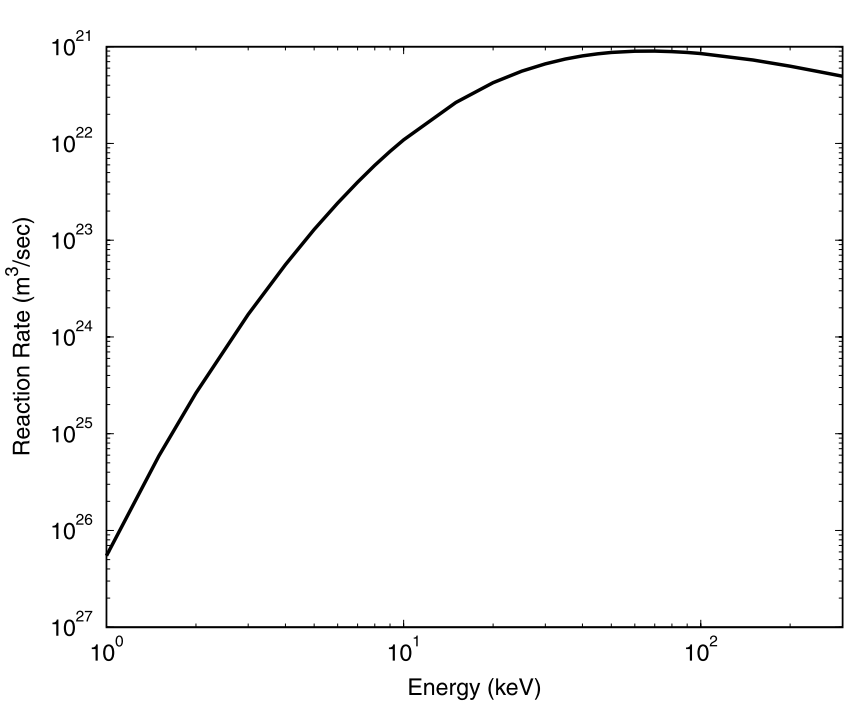

(a)

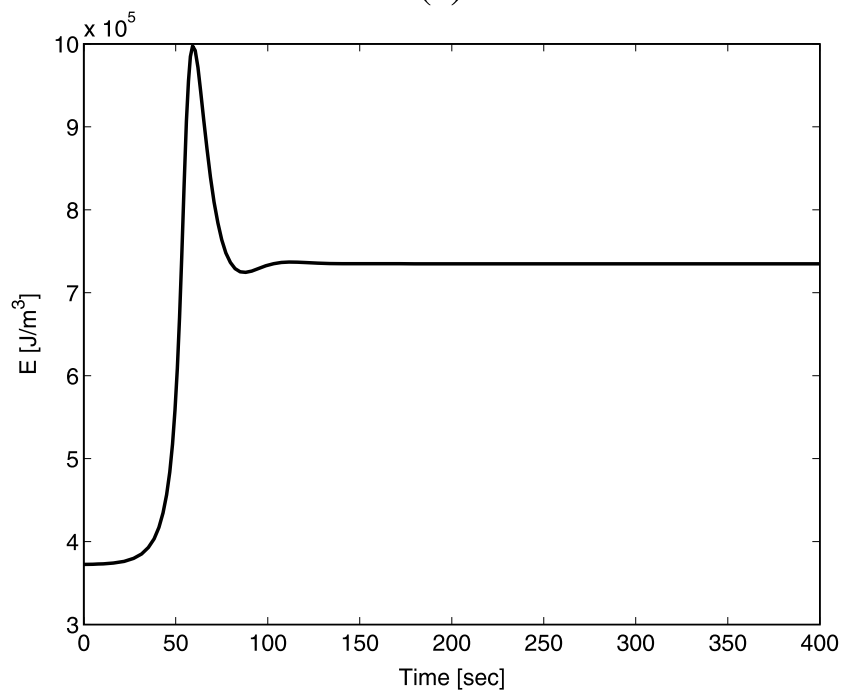

(c)

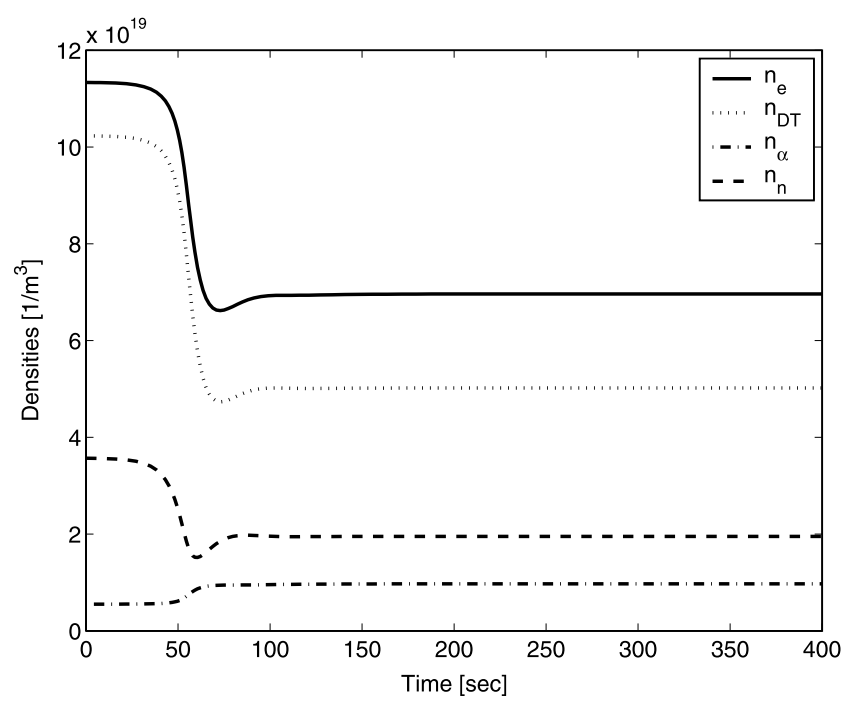

(b)

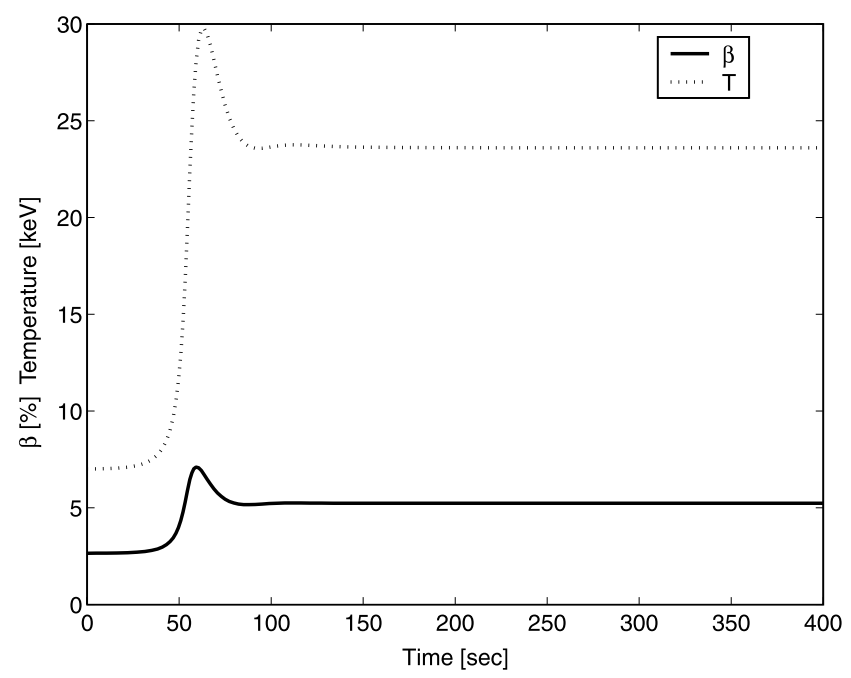

(d)

Fig. 1. Without control, the desired equilibrium is unstable. The result is thermal excursion. MHD stability requirements are violated in this excursion.

working on passive control of the burn condition agree on the necessity of implementing active feedback techniques for the control of the fusion reactor plasma kinetics since this approach has been identified as the most credible control strategy available. ${ }^{1}$

The objective of the controller is to keep the plasma at a desired equilibrium or operating point. The controller must be able to reject perturbations in initial conditions, forcing the plasma back to the equilibrium. Figures $1 \mathrm{~b}, 1 \mathrm{c}$, and $1 \mathrm{~d}$ show the thermokinetically unstable nature of the equilibrium point when no control is present and the system is slightly perturbed. The system leaves the desired equilibrium point (unstable) and settles on a higher temperature equilibrium point (sta- ble). It can be noted how the system is driven from a low-temperature and high-density unstable zone to a high-temperature and low-density stable zone. However, the new equilibrium reached is uneconomical and must be avoided. On the other hand, it is very important to note that the Troyon $\beta$ limit stated in Table I is violated in this thermal excursion.

\section{I.B. Prior Work}

The common denominator of existing works is the approximation of the nonlinear model of the fusion reactor by a linearized one for the purpose of control design. $^{2}$ The nonlinear model is linearized, the controller is 
TABLE I

ITER Machine Parameters*

\begin{tabular}{|lll|}
\hline$I$ & Plasma current & $22.0 \mathrm{MA}$ \\
$R$ & Major radius & $6.0 \mathrm{~m}$ \\
$a$ & Minor radius & $2.15 \mathrm{~m}$ \\
$B$ & Magnetic field & $4.85 \mathrm{~T}$ \\
$\kappa_{\chi}$ & Elongation at $\chi$ & 2.2 \\
$k_{\alpha}$ & Alpha particle confinement & 7 \\
$\quad$ constant & 3 \\
$k_{D T}$ & DT particle confinement & \\
$\quad$ constant & $2.5 I / a B=5.3 \%$ \\
$\beta_{\max }$ & Beta limit & $1100 \mathrm{~m}^{3}$ \\
$V$ & Plasma volume & \\
\hline
\end{tabular}

*See Ref. 29. Although newer ITER configurations are available, this configuration is used only for a simulation purpose to allow the comparison of the nonlinear controller with previous linear controllers. The nonlinear controller synthesis does not depend on the ITER configuration.

synthesized using linear control techniques, and the performance of the resulting linear controller is tested through simulations that use the original nonlinear model. On the other hand, these linear controllers seldom use all the available actuators and typically only one among the actuation concepts (single-input control) is employed in existing works. When tested through nonlinear simulations, these linear controllers succeed in stabilizing the system only against a limited set of perturbations in the initial conditions. To expand operability, we are seeking a systematic procedure for synthesis of burn controllers that are able to stabilize the system against large perturbations in the initial conditions, can work for suppressing thermal excursions as well as for preventing quenches, can operate both at subignition and ignition points, are robust against uncertainties in parameters of the model such as the confinement times of the species, can drive the system from an operating point to another and can change the fusion power during the reactor operation. Such controllers should be based on the full nonlinear model and should make use simultaneously of all the potential actuators. Only those works that use nonmodel based control techniques like neural networks ${ }^{3,4}$ have followed these guidelines. In this work we present a stabilizing controller for the burn condition in fusion reactors synthesized using model-based nonlinear control techniques that avoid the linearization of the model. The avoidance of the linearization allows us to achieve much higher levels of performance and robustness.

Over the years, the physical and technological feasibility of different methods for controlling the burn condition have been studied. ${ }^{5-7}$ In these studies, mainly three different types of actuation have been considered: modulation of auxiliary power, modulation of fueling rate, and controlled injection of impurities.
The controllers based on the modulation of the auxiliary power ${ }^{8-10}$ require operation at subignition points where the auxiliary power is nonzero. As the plasma heats up due to a positive perturbation in the initial temperature, the auxiliary power is reduced by the controller. Since the maximum reduction is complete shutoff of the auxiliary power, there is a limited range of thermal excursions where the control system is effective. The control of negative perturbations in the initial temperature is less demanding and it depends only on the availability of adequate heating capability.

The controllers based on the modulation of the fueling rate ${ }^{11-15}$ allow operation at ignition points where the auxiliary power is zero. However, although they can deal quite well with perturbations in initial conditions leading to thermal excursions, they are not very effective for perturbations in initial conditions leading to quenching.

Controlled introduction of impurities is useful to enhance the radiation losses in the plasma and in this way prevent thermal excursions. For large positive perturbations in the initial temperature this method requires the introduction of a large amount of impurities. Therefore, after controlling the thermal excursion, additional amount of auxiliary power, with the consequent $Q$ reduction, must be provided in order to compensate the radiation losses due to the impurities until they are completely removed from the reactor.

Prior work that combine actuation concepts are Refs. 16, 17, and 18 in 0-D and Refs 19, 20, and 21 in 1 -D models.

\section{I.C. Results of the Paper}

In this work we consider the use of auxiliary power and fueling rate modulations for stabilizing the burn condition of a fusion reactor working at a subignited point against a limited range of perturbations in the initial conditions. However, when we want to work at an ignited point or we want to have the capability of rejecting a larger set of perturbations in the initial conditions, we consider the controlled injection of impurities as an additional actuator.

For burn control purposes there are two principal types of fueling systems: pellet injection and gas puffing. Pellet injection is a better actuator in the sense that its neutral fuel transportation time is shorter. However, it is also technically more complex. This technical aspect of the burn control problem forces us to introduce in our model the effect of the lag due to the diffusion of the fresh fuel into the plasma. A nonlinear backstepping technique is used to handle the lag imposed by the actuator without any kind of further approximation of the model. In this way we make our control system independent of the fueling system and the reactor can be fed either by pellet injection or by gas puffing.

The synthesis of the controller based on the full nonlinear model allows us to deal with a larger set of 
perturbations in initial conditions. The controller can deal simultaneously with perturbations in initial conditions leading to both thermal excursion and quenching, and its effectiveness does not depend on whether the equilibrium operating point is an ignition or a subignition point. Since the nonlinear controller depends parametrically on the equilibrium point, it can drive the system from one equilibrium point to another allowing in this way the change of power, other plasma parameters, like $\beta$ for example, and ignition conditions. Simulation results show good robustness properties against uncertainties in the confinement times. The controller designed ensures setpoint regulation for the energy and plasma parameter $\beta$ with robustness against uncertainties in the confinement times for different species.

\section{I.D. Organization}

In Sec. II we focus on the subignition operation. The model and control objectives are stated and the control laws for the auxiliary power and fueling rate are synthesized for the stabilization of the deviation state variables. The section ends with a presentation of the simulation results. In Sec. III we focus on the ignition operation. In this case we allow the presence of impurities in the fusion reactor. The modifications of the model and the control laws for the auxiliary power and fueling rate are presented together with the synthesis of the control law for the impurity injection. The section is also closed with a computer simulation study and a comparison with previous linear controllers. Finally, the conclusions and some suggestions about future work are presented in Sec. IV. Appendix A states the basics of the Lyapunov stability theory.

\section{SUBIGNITION OPERATION}

\section{II.A. Model}

In this work we use a zero-dimensional model for a fusion reactor which employs approximate particle and energy balance equations. This is fundamentally the same model used by Hui, Fischbach, Bamieh, and Miley. ${ }^{14}$ The alpha-particle balance is given by

$$
\frac{d n_{\alpha}}{d t}=-\frac{n_{\alpha}}{\tau_{\alpha}}+\left(\frac{n_{D T}}{2}\right)^{2}\langle\sigma v\rangle,
$$

where $n_{\alpha}$ and $n_{D T}$ are the alpha and deuterium-tritium (DT) densities, respectively, and $\tau_{\alpha}$ is the confinement time for the alpha particles. This approximate model implies that the $3.52 \mathrm{MeV}$ alpha particles slow down instantaneously, depositing their energy in the flux surface where they are born, which is a reasonable approximation for reactor-size tokamaks. A first order lag is introduced to take into account the diffusion time for neutral fuel atoms to transport into the tokamak core. This lag runs from the start of the fuel injection to the change in deuterium-tritium (DT) ion particle density. The set of equations governing the neutral fuel atom balance and the deuterium-tritium (DT) ionized fuel particle balance is given by

$$
\begin{aligned}
\frac{d n_{D T}}{d t} & =-\frac{n_{D T}}{\tau_{D T}}-2\left(\frac{n_{D T}}{2}\right)^{2}\langle\sigma v\rangle+\frac{n_{n}}{\tau_{d}} \\
\frac{d n_{n}}{d t} & =-\frac{n_{n}}{\tau_{d}}+S,
\end{aligned}
$$

where $n_{n}$ is the neutral fuel density, defined as the number of neutral fuel atoms divided by the core volume, $S$ (input) is the refueling rate $(50: 50 \mathrm{D}-\mathrm{T})$, defined as the number of neutral fuel atoms injected per unit time divided by the core volume, $\tau_{D T}$ is the confinement time for the ionized fuel particles, and $\tau_{d}$ is the controller lag time. The energy balance is given by

$$
\begin{aligned}
\frac{d E}{d t}= & -\frac{E}{\tau_{E}}+\left(\frac{n_{D T}}{2}\right)^{2}\langle\sigma v\rangle Q_{\alpha}-P_{\text {rad }}+P_{a u x} \\
& +P_{\text {ohmic }},
\end{aligned}
$$

where $E$ is the plasma energy, $\tau_{E}$ is the energy confinement time, $Q_{\alpha}=3.52 \mathrm{MeV}$ is the energy of the alpha particles, $P_{\text {aux }}$ (input) is the auxiliary power, the radiation loss $P_{\text {rad }}$ is given by

$$
P_{\text {rad }}=P_{\text {brem }}=A_{b} Z_{\text {eff }} n_{e}^{2} \sqrt{T}
$$

where $A_{b}=4.85 \times 10^{-37} \mathrm{Wm}^{3} / \sqrt{\mathrm{keV}}$ is the bremsstrahlung radiation coefficient, and the ohmic power $P_{\text {ohmic }}$ is written as

$$
P_{\text {ohmic }}=\eta j^{2}
$$

where $\eta$ is the Spitzer resistivity and $j$ is the plasma current density. The DT reactivity $\langle\sigma v\rangle$, shown in Fig. 1a, is a highly nonlinear, positive, and bounded function of the plasma temperature $T$ given by

$$
\langle\sigma v\rangle=\exp \left(\frac{a_{1}}{T^{r}}+a_{2}+a_{3} T+a_{4} T^{2}+a_{5} T^{3}+a_{6} T^{4}\right),
$$

and its parameters $a_{i}$ and $r$ are taken from Ref. 22 .

No explicit evolution equation is provided for the electron density $n_{e}$ since we can obtain it from the neutrality condition $n_{e}=n_{D T}+2 n_{\alpha}$, whereas the effective atomic number, the total density and the energy are written as 


$$
\begin{gathered}
Z_{\text {eff }}=\frac{\sum_{i} n_{i} Z_{i}^{2}}{n_{e}}=\frac{n_{D T}+4 n_{\alpha}}{n_{e}}, \\
n=n_{\alpha}+n_{D T}+n_{e}=2 n_{D T}+3 n_{\alpha},
\end{gathered}
$$

and

$$
E=\frac{3}{2} n T \Rightarrow T=\frac{2}{3} \frac{E}{2 n_{D T}+3 n_{\alpha}},
$$

where $Z_{i}$ is the atomic number of the different ions. The energy confinement scaling used in this work is ITER90H-P (Ref. 23) because it allows the comparison with previous linear controllers based on this scaling. However, it will be clear from the synthesis procedure that the results can be extended to newer scalings. This scales with plasma parameters as

$$
\tau_{E}=f 0.082 I^{1.02} R^{1.6} B^{0.15} A_{i}^{0.5} \kappa_{\chi}^{-0.19} P^{-0.47}=k P^{-0.47},
$$

where the isotopic number $A_{i}$ is 2.5 for the 50:50 DT mixture, the ITER machine parameters are defined in Table I, and the factor scale $f$ depends on the confinement mode. The isotopic number, factor scale, and ITER machine parameters can be rewritten as a constant $k$ because it is assumed that a magnetic controller is regulating these variables. The net plasma heating power $P$ is defined as

$$
\begin{aligned}
P= & \left(\frac{n_{D T}}{2}\right)^{2}\langle\sigma v\rangle Q_{\alpha}-A_{b}\left(n_{D T}+4 n_{\alpha}\right)\left(n_{D T}+2 n_{\alpha}\right) \\
& \times \sqrt{\frac{2}{3} \frac{E}{2 n_{D T}+3 n_{\alpha}}}+P_{a u x}+\eta j^{2} \\
= & \text { alpha heating }-P_{\text {rad }}+P_{\text {aux }}+P_{\text {ohmic }}
\end{aligned}
$$

The net plasma heating power is compared with the $\mathrm{L}-\mathrm{H}$ transition power (threshold power) $P_{t h r}$ to determine the value of $f$. This threshold power can be written as ${ }^{24}$

$$
P_{t h r}=2.84 M^{-1} B^{0.82} n_{e}^{0.58} R^{1.00} a^{0.81},
$$

where the units are amu, T, $10^{20} \mathrm{~m}^{-3}$, and $\mathrm{m}$. The confinement times for the different species are scaled with the energy confinement time $\tau_{E}$ as

$$
\tau_{\alpha}=k_{\alpha} \tau_{E}, \quad \tau_{D T}=k_{D T} \tau_{E}
$$

We are interested in studying controller performance as a ratio of the lag time $\tau_{d}$ to the energy confinement time
$\tau_{E}$. For this reason and the fact that this lag time could be sizable, of the order of the confinement time, we also scale it with the energy confinement time $\tau_{E}$ as $\tau_{d}=$ $k_{d} \tau_{E}$. We address the most difficult case by assuming $\tau_{d}$ as a function of the states, even though the lag time, being mainly an actuator parameter, should not depend on the internal states. For simulation purposes we use $k_{d}=1$ which represents an appreciable lag in the actuation. However, the controller succeeds in dealing with this lag.

\section{II.B. Control Objective}

The possible operating points of the reactor are given by the equilibria of the dynamic equations. The density state variables $\bar{n}_{\alpha}, \bar{n}_{D T}, \bar{n}_{n}$, energy state variable $\bar{E}$ and inputs $\bar{P}_{\text {aux }}, \bar{S}$ at the equilibrium, are calculated as solutions of the nonlinear algebraic equations obtained by setting the left sides in Eqs. (1) through (4) to zero when two of the plasma parameters such as $T$ and $\beta$, for example, are chosen arbitrarily:

$$
\begin{aligned}
& 0=-\frac{\bar{n}_{\alpha}}{\bar{\tau}_{\alpha}}+\left(\frac{\bar{n}_{D T}}{2}\right)^{2}\langle\overline{\sigma v}\rangle, \\
& 0=-\frac{\bar{n}_{D T}}{\bar{\tau}_{D T}}-2\left(\frac{\bar{n}_{D T}}{2}\right)^{2}\langle\overline{\sigma v}\rangle+\frac{\bar{n}_{n}}{\bar{\tau}_{d}}, \\
& 0=-\frac{\bar{n}_{n}}{\bar{\tau}_{d}}+\bar{S},
\end{aligned}
$$

and

$$
0=-\frac{\bar{E}}{\bar{\tau}_{E}}+\bar{P},
$$

where

$$
\begin{aligned}
\bar{P}= & \left(\frac{\bar{n}_{D T}}{2}\right)^{2}\langle\overline{\sigma v}\rangle Q_{\alpha}-A_{b}\left(\bar{n}_{D T}+4 \bar{n}_{\alpha}\right)\left(\bar{n}_{D T}+2 \bar{n}_{\alpha}\right) \\
& \times \sqrt{\frac{2}{3} \frac{\bar{E}}{2 \bar{n}_{D T}+3 \bar{n}_{\alpha}}}+\bar{P}_{a u x}+\bar{\eta} j^{2} .
\end{aligned}
$$

Defining the deviations from the desired equilibrium values as $\tilde{n}_{\alpha}=n_{\alpha}-\bar{n}_{\alpha}, \tilde{n}_{D T}=n_{D T}-\bar{n}_{D T}, \tilde{n}_{n}=n_{n}-$ $\bar{n}_{n}, \widetilde{E}=E-\bar{E}, \widetilde{P}_{a u x}=P_{a u x}-\bar{P}_{a u x}$, and $\tilde{S}=S-\bar{S}$, we write the dynamic equations for the deviations as

$$
\begin{aligned}
\frac{d \tilde{n}_{\alpha}}{d t}= & -\frac{\tilde{n}_{\alpha}}{\tau_{\alpha}}+\left(\frac{\tilde{n}_{D T}}{2}\right)^{2}\langle\sigma v\rangle+\frac{1}{2} \tilde{n}_{D T} \bar{n}_{D T}\langle\sigma v\rangle \\
& +u_{\alpha}
\end{aligned}
$$




$$
\begin{aligned}
\frac{d \tilde{n}_{D T}}{d t}= & -\frac{\tilde{n}_{D T}}{\tau_{D T}}-2\left(\frac{\tilde{n}_{D T}}{2}\right)^{2}\langle\sigma v\rangle-\tilde{n}_{D T} \bar{n}_{D T}\langle\sigma v\rangle \\
& +\frac{\tilde{n}_{n}}{\tau_{d}}+u_{D T},
\end{aligned}
$$$$
\frac{d \tilde{n}_{n}}{d t}=\hat{S}
$$

and

$$
\frac{d \widetilde{E}}{d t}=-\frac{\widetilde{E}}{\tau_{E}}-\left[\frac{\bar{E}}{\tau_{E}}-\left[\left(\frac{n_{D T}}{2}\right)^{2}\langle\sigma v\rangle Q_{\alpha}+\eta j^{2}+u\right]\right],
$$

where

$$
\begin{gathered}
u_{\alpha}=-\frac{\bar{n}_{\alpha}}{\tau_{\alpha}}+\left(\frac{\bar{n}_{D T}}{2}\right)^{2}\langle\sigma v\rangle, \\
u_{D T}=-\frac{\bar{n}_{D T}}{\tau_{D T}}-2\left(\frac{\bar{n}_{D T}}{2}\right)^{2}\langle\sigma v\rangle+\frac{\bar{n}_{n}}{\tau_{d}}, \\
\hat{S}=-\frac{\tilde{n}_{n}}{\tau_{d}}+\tilde{S}-\frac{\bar{n}_{n}}{\tau_{d}}+\bar{S}=-\frac{n_{n}}{\tau_{d}}+S,
\end{gathered}
$$

and

$$
\begin{aligned}
u= & P_{\text {aux }}-P_{\text {rad }}=P_{\text {aux }}-A_{b}\left(n_{D T}+4 n_{\alpha}\right)\left(n_{D T}+2 n_{\alpha}\right) \\
& \times \sqrt{\frac{2}{3} \frac{E}{2 n_{D T}+3 n_{\alpha}}} .
\end{aligned}
$$

The control objective is to drive the initial perturbations in $\tilde{n}_{\alpha}, \tilde{n}_{D T}, \tilde{n}_{n}$, and $\widetilde{E}$ to zero using actuation through $P_{a u x}$ and $S$. All the states are assumed to be available for feedback, either by measurement or by estimation.

\section{II.C. Controller Design}

We start by looking for a control which stabilizes $\widetilde{E}$. We choose $u$ in order to reduce Eq. (22) to

$$
\frac{d \widetilde{E}}{d t}=-K_{E} \frac{\widetilde{E}}{\tau_{E}}
$$

with $K_{E}>0$. This means, after replacing $u$ by its expression, that we choose $P_{\text {aux }}$ such that

FUSION SCIENCE AND TECHNOLOGY

VOL. 43 JAN. 2003

$$
\begin{aligned}
-K_{E} \frac{\widetilde{E}}{\tau_{E}}= & -\frac{\widetilde{E}}{\tau_{E}}-\frac{\bar{E}}{\tau_{E}}+\left(\frac{n_{D T}}{2}\right)^{2}\langle\sigma v\rangle Q_{\alpha}+\eta j^{2} \\
& -A_{b}\left(n_{D T}+4 n_{\alpha}\right)\left(n_{D T}+2 n_{\alpha}\right) \\
& \times \sqrt{\frac{2}{3} \frac{E}{2 n_{D T}+3 n_{\alpha}}}+P_{\text {aux }}
\end{aligned}
$$

which in turn gives

$$
\begin{aligned}
P_{a u x}= & \frac{\bar{E}}{\tau_{E}}-\left(\frac{n_{D T}}{2}\right)^{2}\langle\sigma v\rangle Q_{\alpha}-\eta j^{2} \\
& +A_{b}\left(n_{D T}+4 n_{\alpha}\right)\left(n_{D T}+2 n_{\alpha}\right) \\
& \times \sqrt{\frac{2}{3} \frac{E}{2 n_{D T}+3 n_{\alpha}}} \\
& +\left(1-K_{E}\right) \frac{\tilde{E}}{\tau_{E}} .
\end{aligned}
$$

The gain $K_{E}$ allows us to regulate the response rate of $\widetilde{E}$. This helps us to regulate the control force in order to keep the modulation rate of the auxiliary power in $\mathrm{MW} / \mathrm{s}$ below the technological limits. Nevertheless the computation of $P_{a u x}$ from Eq. (28) is not direct because $\tau_{E}$ is a function of $P_{a u x}$. In order to simplify the computation of $P_{\text {aux }}$ we choose $K_{E}=1$, i.e., we choose $u$ such that

$$
\frac{\bar{E}}{\tau_{E}}-\left[\left(\frac{n_{D T}}{2}\right)^{2}\langle\sigma v\rangle Q_{\alpha}+\eta j^{2}+u\right]=0 .
$$

This means, after replacing $u$ by its expression, that we choose $P_{\text {aux }}$ such that

$$
\begin{aligned}
\frac{\bar{E}}{\tau_{E}}= & \left(\frac{n_{D T}}{2}\right)^{2}\langle\sigma v\rangle Q_{\alpha}+\eta j^{2} \\
& -A_{b}\left(n_{D T}+4 n_{\alpha}\right)\left(n_{D T}+2 n_{\alpha}\right) \\
& \times \sqrt{\frac{2}{3} \frac{E}{2 n_{D T}+3 n_{\alpha}}}+P_{a u x}=P .
\end{aligned}
$$

From the equilibrium Eq. (18) for the energy and the correlation between the energy confinement scaling $\tau_{E}$ and the power $P$ given by Eq. (11), we realize that the solution for Eq. (30) is $P=\bar{P}$. Therefore, the control strategy will be to adjust $P_{\text {aux }}$ to make $P$ constant and equal to $\bar{P}$ satisfying Eq. (30) and reducing Eq. (22) to

$$
\frac{d \widetilde{E}}{d t}=-\frac{\widetilde{E}}{\tau_{E}} .
$$


The subsystem $\widetilde{E}$ is exponential stable since $\tau_{E}>0$. The controller that implements Eq. (30) is synthesized as

$$
\begin{aligned}
P_{\text {aux }}= & \bar{P}-\left(\frac{n_{D T}}{2}\right)^{2}\langle\sigma v\rangle Q_{\alpha}-\eta j^{2} \\
& +A_{b}\left(n_{D T}+4 n_{\alpha}\right)\left(n_{D T}+2 n_{\alpha}\right) \sqrt{\frac{2}{3} \frac{E}{2 n_{D T}+3 n_{\alpha}}} .
\end{aligned}
$$

In practice, there are limitations on the power supply system that constrain the rate at which the auxiliary power can be varied. This condition can be written

$$
\left|\frac{d P_{\text {aux }}}{d t}\right|<R_{\text {aux }},
$$

where $R_{\text {aux }}$ is the maximum auxiliary power ramp rate. It is thought that $R_{\text {aux }} \sim 10-20 \mathrm{MW} / \mathrm{s}$ may be achievable for ITER (Ref. 6).

After stabilizing $\widetilde{E}$ using $P_{a u x}$ as controller, we must focus in Eqs. (19) and (20) to achieve stability for $\tilde{n}_{D T}$ and $\tilde{n}_{\alpha}$. This controller not only stabilizes $\widetilde{E}$ but also makes $P=\bar{P}$. Then we have $\tau_{E}=\bar{\tau}_{E}, \tau_{\alpha}=\bar{\tau}_{\alpha}, \tau_{D T}=\bar{\tau}_{D T}$, and $\tau_{d}=\bar{\tau}_{d}$. The equations for the subsystem $\left(\tilde{n}_{D T}, \tilde{n}_{n}\right)$ can be rewritten as

$$
\begin{aligned}
\frac{d \tilde{n}_{D T}}{d t}= & -\frac{\tilde{n}_{D T}}{\bar{\tau}_{D T}}-2\left(\frac{\tilde{n}_{D T}}{2}\right)^{2}\langle\sigma v\rangle-\tilde{n}_{D T} \bar{n}_{D T}\langle\sigma v\rangle \\
& +\frac{\tilde{n}_{n}}{\bar{\tau}_{d}}+u_{D T}^{*}
\end{aligned}
$$

and

$$
\frac{d \tilde{n}_{n}}{d t}=S^{*}
$$

where

$$
u_{D T}^{*}=-\frac{\bar{n}_{D T}}{\bar{\tau}_{D T}}-2\left(\frac{\bar{n}_{D T}}{2}\right)^{2}\langle\sigma v\rangle+\frac{\bar{n}_{n}}{\bar{\tau}_{d}}
$$

and

$$
S^{*}=-\frac{n_{n}}{\bar{\tau}_{d}}+S
$$

A nonlinear backstepping technique is used for the stabilization of the $\left(\tilde{n}_{D T}, \tilde{n}_{n}\right)$ subsystem. With this purpose we take $\tilde{n}_{n}$ as the virtual control $w$,

$$
\begin{aligned}
\frac{d \tilde{n}_{D T}}{d t}= & -\left[\frac{1}{\bar{\tau}_{D T}}+\bar{n}_{D T}\langle\sigma v\rangle\right] \tilde{n}_{D T}-2\left(\frac{\tilde{n}_{D T}}{2}\right)^{2}\langle\sigma v\rangle \\
& +u_{D T}^{*}+\frac{w}{\bar{\tau}_{d}} .
\end{aligned}
$$

Since $\left[1 / \bar{\tau}_{D T}+\bar{n}_{D T}\langle\sigma v\rangle\right]$ is positive, we exponentially stabilize $\tilde{n}_{D T}$ taking

$w=\alpha\left(n_{\alpha}, n_{D T}, E\right)=\bar{\tau}_{d}\left[2\left(\frac{\tilde{n}_{D T}}{2}\right)^{2}\langle\sigma v\rangle-u_{D T}^{*}\right]$,

reducing in this way Eq. (33) to:

$$
\frac{d \tilde{n}_{D T}}{d t}=-\left[\frac{1}{\bar{\tau}_{D T}}+\bar{n}_{D T}\langle\sigma v\rangle\right] \tilde{n}_{D T} .
$$

Defining now $z=\tilde{n}_{n}-\alpha \Leftrightarrow \tilde{n}_{n}=z+\alpha$, we can write

$$
\dot{z}=\dot{\tilde{n}}_{n}-\dot{\alpha}=S^{*}-\dot{\alpha}
$$

where $\left({ }^{\cdot}\right)=(d / d t)(\quad)$. Taking the Lyapunov function (see the Appendix and Ref. 25) candidate $V=\left(\tilde{n}_{D T}^{2} / 2\right)+$ $\left(z^{2} / 2\right)$, from Eqs. (33) and (38) and taking into account our definition (37) for $\alpha$ we can compute

$$
\begin{aligned}
& \dot{V}= \tilde{n}_{D T} \dot{\tilde{n}}_{D T}+z \dot{z} \\
&=\tilde{n}_{D T}[-\left(\frac{1}{\bar{\tau}_{D T}}+\bar{n}_{D T}\langle\sigma v\rangle\right) \tilde{n}_{D T}-2\left(\frac{\tilde{n}_{D T}}{2}\right)^{2}\langle\sigma v\rangle \\
&\left.\quad+u_{D T}^{*}+\frac{\alpha+z}{\bar{\tau}_{d}}\right]+z\left[S^{*}-\dot{\alpha}\right] \\
&=-\left[\frac{1}{\bar{\tau}_{D T}}+\bar{n}_{D T}\langle\sigma v\rangle\right] \tilde{n}_{D T}^{2}+z\left[S^{*}-\dot{\alpha}+\frac{\tilde{n}_{D T}}{\bar{\tau}_{d}}\right] .
\end{aligned}
$$

Taking

$$
\begin{aligned}
S^{*} & =-K_{S} z+\dot{\alpha}-\frac{\tilde{n}_{D T}}{\bar{\tau}_{d}} \\
& =-K_{S}\left(\tilde{n}_{n}-\alpha\right)+\dot{\alpha}-\frac{\tilde{n}_{D T}}{\bar{\tau}_{d}} \\
S & =-K_{S}\left(\tilde{n}_{n}-\alpha\right)+\dot{\alpha}-\frac{\tilde{n}_{D T}}{\bar{\tau}_{d}}+\frac{n_{n}}{\bar{\tau}_{d}},
\end{aligned}
$$

with $K_{S}>0$, we have

$$
\dot{V}=-\left[\frac{1}{\bar{\tau}_{D T}}+\bar{n}_{D T}\langle\sigma v\rangle\right] \tilde{n}_{D T}^{2}-K_{S} z^{2}<0,
$$

and we achieve exponential stability for the subsystem $\left(\tilde{n}_{D T}, \tilde{n}_{n}\right)$.

Rewriting the equation for $\alpha$, taking into account the expression for $u_{D T}^{*}$ from Eq. (35), as

$$
\begin{aligned}
\alpha\left(n_{\alpha}, n_{D T}, E\right)=\bar{\tau}_{d} & {\left[2\left(\frac{\tilde{n}_{D T}}{2}\right)^{2}\langle\sigma v\rangle+\frac{\bar{n}_{D T}}{\bar{\tau}_{D T}}\right.} \\
& \left.+2\left(\frac{\bar{n}_{D T}}{2}\right)^{2}\langle\sigma v\rangle-\frac{\bar{n}_{n}}{\bar{\tau}_{d}}\right],
\end{aligned}
$$


we can compute

$$
\begin{gathered}
\dot{\alpha}=\bar{\tau}_{d}\left[\langle\sigma v\rangle \tilde{n}_{D T} \dot{\tilde{n}}_{D T}+2\left(\frac{\tilde{n}_{D T}}{2}\right)^{2}\langle\sigma \dot{v}\rangle\right. \\
\left.+2\left(\frac{\bar{n}_{D T}}{2}\right)^{2}\langle\dot{\sigma} v\rangle\right],
\end{gathered}
$$

where

$$
\begin{aligned}
\langle\sigma \dot{v}\rangle & =\frac{d\langle\sigma v\rangle}{d T} \dot{T} \\
& =\frac{d\langle\sigma v\rangle}{d T}\left[\frac{\partial T}{\partial E} \dot{E}+\frac{\partial T}{\partial n_{D T}} \dot{n}_{D T}+\frac{\partial T}{\partial n_{\alpha}} \dot{n}_{\alpha}\right]
\end{aligned}
$$

and we compute

$$
\begin{aligned}
\frac{d\langle\sigma v\rangle}{d T}= & \langle\sigma v\rangle \\
& \times\left(\frac{-a_{1} r}{T^{r+1}}+a_{3}+2 a_{4} T+3 a_{5} T^{2}+4 a_{6} T^{3}\right),
\end{aligned}
$$

from Eq. (7) and

$$
\begin{aligned}
\frac{\partial T}{\partial E} & =\frac{2}{3} \frac{1}{2 n_{D T}+3 n_{\alpha}}, \quad \frac{\partial T}{\partial n_{D T}}=-\frac{4}{3} \frac{E}{\left(2 n_{D T}+3 n_{\alpha}\right)^{2}}, \\
\frac{\partial T}{\partial n_{\alpha}} & =-2 \frac{E}{\left(2 n_{D T}+3 n_{\alpha}\right)^{2}}
\end{aligned}
$$

from Eq. (10). The expressions for $\dot{n}_{\alpha}, \dot{n}_{D T}$, and $\dot{E}$ can be obtained from Eqs. (1), (2), and (4) respectively.

In order to finish our stability analysis we rewrite here the equation for $\tilde{n}_{\alpha}$ taking into account the fact that $\tau_{\alpha}=\bar{\tau}_{\alpha}$,

$$
\begin{aligned}
\frac{d \tilde{n}_{\alpha}}{d t} & =-\frac{\tilde{n}_{\alpha}}{\bar{\tau}_{\alpha}}+\left(\frac{\tilde{n}_{D T}}{2}\right)^{2}\langle\sigma v\rangle+\frac{1}{2} \tilde{n}_{D T} \bar{n}_{D T}\langle\sigma v\rangle+u_{\alpha}^{*} \\
u_{\alpha}^{*} & =-\frac{\bar{n}_{\alpha}}{\bar{\tau}_{\alpha}}+\left(\frac{\bar{n}_{D T}}{2}\right)^{2}\langle\sigma v\rangle .
\end{aligned}
$$

We note that $\tilde{n}_{\alpha}$ is ISS (input-state stable) (see Ref. 25, section 5.3) with respect to $\tilde{n}_{D T}$ and $u_{\alpha}^{*}$. This means that the state $\tilde{n}_{\alpha}$ will be bounded if the inputs $\tilde{n}_{D T}$ and $u_{\alpha}^{*}$ are bounded. Since $\tilde{n}_{D T}$ is bounded (because it is exponentially stable) and $u_{\alpha}^{*}$ is bounded (because $\widetilde{E}$ is exponentially stable and $\langle\sigma v\rangle$ is a bounded function), $\tilde{n}_{\alpha}$ will be bounded for all time. In addition, once $E$ converges to $\bar{E}$ $(\widetilde{E} \rightarrow 0)$ and $n_{D T}$ converges to $\bar{n}_{D T}\left(\tilde{n}_{D T} \rightarrow 0\right)$ this equation reduces to

$$
\frac{d \tilde{n}_{\alpha}}{d t}=-\frac{\tilde{n}_{\alpha}}{\bar{\tau}_{\alpha}}+u_{\alpha}^{*} .
$$

Recalling Eq. (15), we take into consideration that

$$
-\frac{\bar{n}_{\alpha}}{\bar{\tau}_{\alpha}}+\left(\frac{\bar{n}_{D T}}{2}\right)^{2}\langle\overline{\sigma v}\rangle=0 .
$$

In addition the function $\langle\sigma v\rangle$ is a function of $T=\frac{2}{3}[\bar{E} /$ $\left.\left(2 \bar{n}_{D T}+3 n_{\alpha}\right)\right]$ and has a positive derivative in the region of interest. This means that for a positive deviation $\tilde{n}_{\alpha}$, $n_{\alpha}$ increases, $T$ decreases, $\langle\sigma v\rangle$ decreases, and $u_{\alpha}^{*}$ becomes negative. On the other hand, for a negative deviation $\tilde{n}_{\alpha}, n_{\alpha}$ decreases, $T$ increases, $\langle\sigma v\rangle$ increases and $u_{\alpha}^{*}$ becomes positive. Consequently $u_{\alpha}^{*}$ has the same sign as $-\left(\tilde{n}_{\alpha} / \bar{\tau}_{\alpha}\right)$ and vanishes when $\tilde{n}_{\alpha}$ vanishes $(\langle\sigma v\rangle=$ $\langle\overline{\sigma v}\rangle)$. This allows us to conclude exponential stability for $\tilde{n}_{\alpha}$.

We have shown that the control laws (31) and (39) stabilize the system exponentially. However this is true as long as condition (30) can be satisfied with $P_{\text {aux }}>0$. As the plasma heats up due to a positive perturbation in the initial temperature, the auxiliary power is reduced by the controller. Since the maximum reduction is complete shutoff of the auxiliary power, there is a maximum size of thermal excursions for which the control system is effective. This maximum depends on the value of $\bar{P}_{a u x}$. Hence, we have a trade-off between the size of the maximum positive perturbation in the initial temperature that can be rejected by the controller and $Q$ at the working point. The controlled injection of impurities will help to overcome this limitation.

\section{II.D. Simulation Results}

In this section we show, through a simulation study, the performance of the controller stabilizing the equilibrium point characterized by those values given in Table II. For all the simulations presented here, the controller gains $K_{S}=0.1$ and $K_{E}=1$, a maximum auxiliary power ramp rate $R_{\text {aux }}=10 \mathrm{MW} / \mathrm{s}$ (worst case), and a scale factor $f=f^{H}=0.85$ for the energy confinement time (11) have been used. When $P<P_{t h r}$ we adopt $f=f^{L}=f^{H} / 2$.

Figure 2 confirms that this controller can, without any saturation, reject positive perturbations of up to $10 \%$

\section{TABLE II}

ITER Equilibrium Point 1-Subignition Point

\begin{tabular}{|lll|}
\hline $\bar{T}$ & Temperature & $6 \mathrm{keV}$ \\
$\bar{n}_{e}$ & Electron density & $1.49 \times 10^{20} \mathrm{~m}^{-3}$ \\
$\bar{f}_{\alpha}$ & Alpha fraction & $3.69 \%$ \\
$\bar{\beta}$ & Beta & $3 \%$ \\
$\bar{n}_{\alpha}$ & Alpha density & $5.49 \times 10^{18} \mathrm{~m}^{-3}$ \\
$\bar{n}_{D T}$ & DT density & $1.38 \times 10^{20} \mathrm{~m}^{-3}$ \\
$\bar{E}$ & Energy & $4.21 \times 10^{5} \mathrm{~J} \cdot \mathrm{m}^{-3}$ \\
$\bar{P}_{\text {aux }}$ & Auxiliary power & $1.84 \times 10^{4} \mathrm{~W} \cdot \mathrm{m}^{-3}$ \\
$\bar{S}$ & Fuel rate & $6.98 \times 10^{18} \mathrm{~m}^{-3} \cdot \mathrm{s}^{-1}$ \\
\hline
\end{tabular}



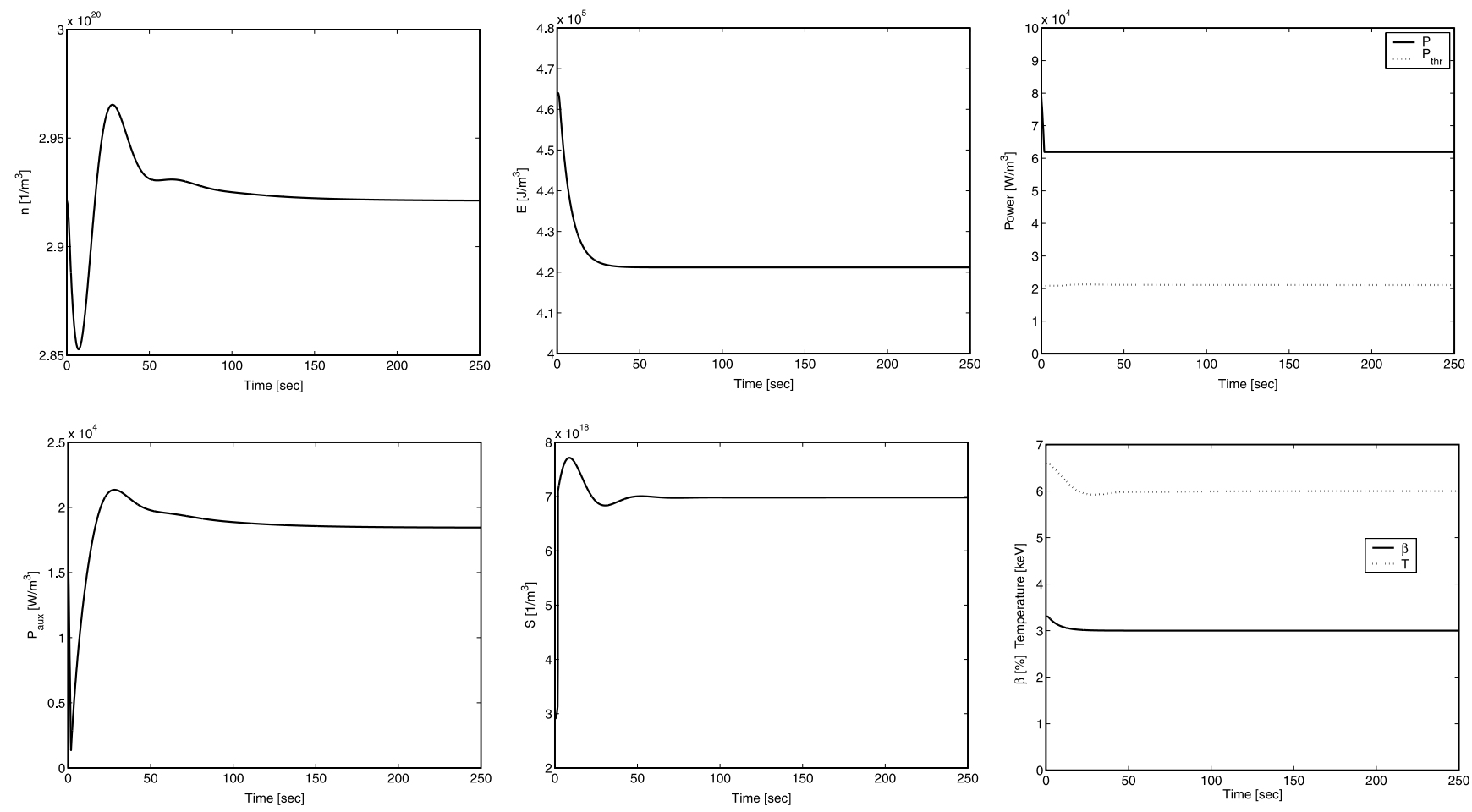

Fig. 2. With control, under initial perturbation of $10 \%$ in $T, 20 \%$ in $f_{\alpha}=n_{\alpha} / n_{e}$ and $0 \%$ in $n$, the system returns to the desired equilibrium $\left(K_{S}=0.1, K_{E}=1\right) . \bar{P}_{a u x}$ is big enough to ensure $P$ to be constant.

in the initial condition of the plasma temperature $T$. Bromberg, Fisher, and Cohn showed in Ref. 8, using linear analysis, that for given power multiplication $Q$ and ion temperature, there is a limited range of perturbations in the initial condition of $T$ where the control system based only on the auxiliary power modulation is effective. They estimated that $Q$ should be less than 17 for stabilizing a $10 \%$ perturbation in plasma temperature for a reactor operating at a central ion temperature of $15 \mathrm{keV}$. However, it is also stated in their work that when the reactor operates at lower temperatures, the maximum value of $Q$ allowed for stabilizing the same size of perturbation is dramatically reduced. In this case we are working at an equilibrium point characterized by $Q=17.63$ and a low temperature $T=6 \mathrm{keV}$ and the controller designed is still able to reject $17.5 \%$ perturbations in the plasma temperature. Although $\bar{P}_{\text {aux }}$ is big enough to ensure $P$ to be constant, this is not achieved perfectly due to the limit in the rate of change of the auxiliary power $P_{\text {aux }}$. However this is the only effect of $R_{\text {aux }}$ and no loss of stabilization capability is found through extensive simulation study. If $R_{\text {aux }}$ were higher, we would have $P=\bar{P}$ for all time which is the characteristic of this controller. Precisely for this characteristic it is very hard to see the net plasma power $P$ drop below the threshold power $P_{t h r}$.

Figure 3 shows us that the control of negative perturbations in the initial condition of $T$ is only limited by the capability of the auxiliary power. Assuming there is no saturation of the actuator, the controller is capable of rejecting a $-50 \%$ perturbation in plasma temperature. The effect of the maximum auxiliary power ramp rate can be appreciated in the evolution of the auxiliary power $P_{\text {aux }}$. Nevertheless, the controller is still able to stabilize the system.

\section{IGNITION OPERATION}

\section{III.A. Model}

In order to stabilize the fusion reactor working at an ignition point or to reject large perturbations in the initial conditions when working at a subignition point, we are going to allow the presence of impurities. Although the title of the section makes direct reference to ignition operation points, the approach and results are also valid for those subignition operation points that cannot be stabilized only by the modulation of auxiliary power. The model is modified here modeling the power losses due to the line and recombination radiations in addition to the bremsstrahlung radiation. In this way we are not restricted to work only with low $Z$ impurities.

Consequently, the zero-dimensional model given by Eqs. (1) through (4) is now modified and enlarged to adopt the following form: 

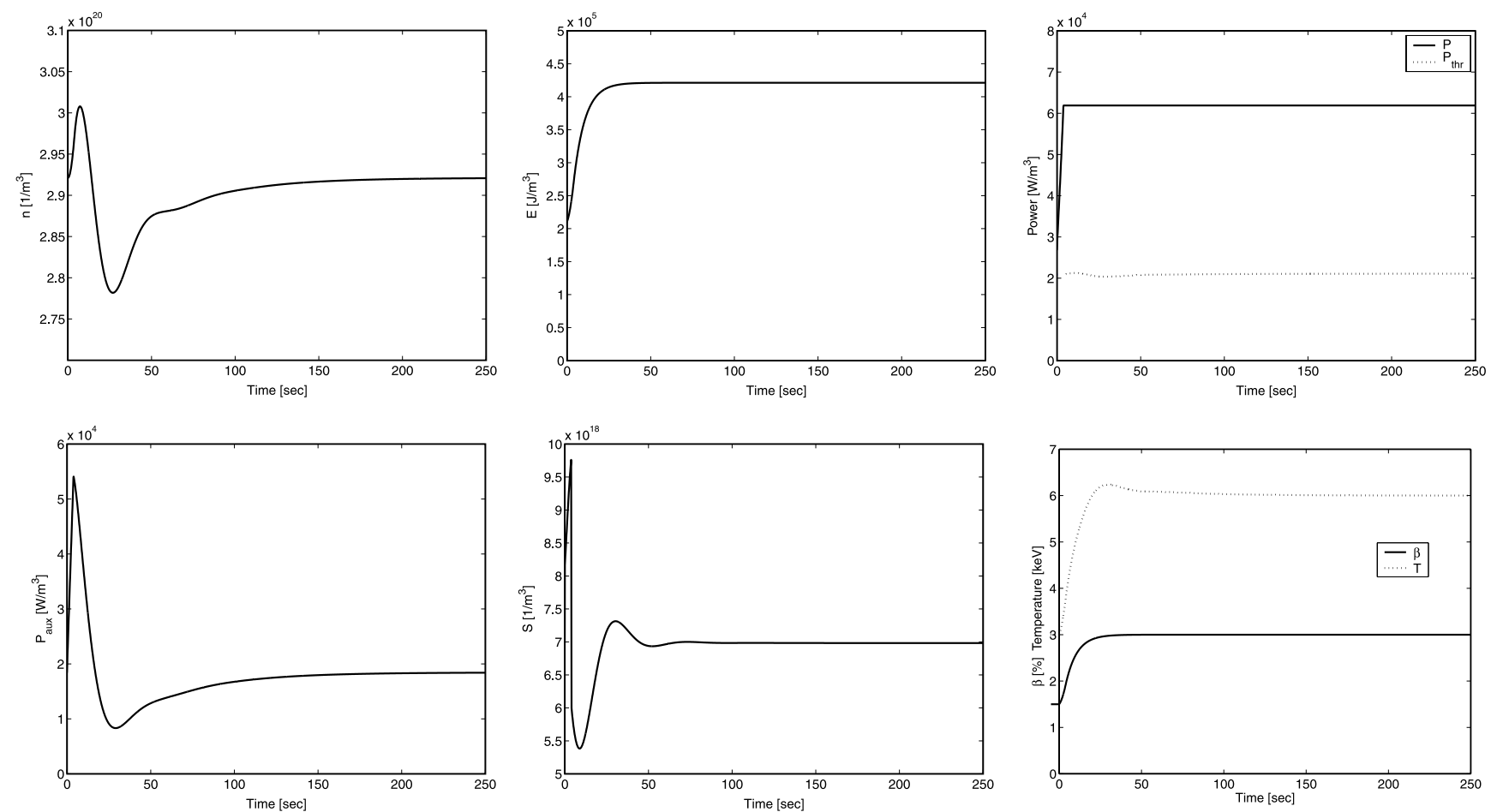

Fig. 3. With control, under initial perturbation of $-50 \%$ in $T,-85 \%$ in $f_{\alpha}=n_{\alpha} / n_{e}$ and $0 \%$ in $n$, the system returns to the desired equilibrium $\left(K_{S}=0.1, K_{E}=1\right)$. In this case, the size of the negative perturbation in the initial condition that can be rejected depends mainly on the available $P_{\text {aux }}$.

$$
\begin{aligned}
\frac{d n_{\alpha}}{d t} & =-\frac{n_{\alpha}}{\tau_{\alpha}}+\left(\frac{n_{D T}}{2}\right)^{2}\langle\sigma v\rangle, \\
\frac{d n_{D T}}{d t} & =-\frac{n_{D T}}{\tau_{D T}}-2\left(\frac{n_{D T}}{2}\right)^{2}\langle\sigma v\rangle+\frac{n_{n}}{\tau_{d}}, \\
\frac{d n_{n}}{d t} & =-\frac{n_{n}}{\tau_{d}}+S, \\
\frac{d n_{I}}{d t} & =-\frac{n_{I}}{\tau_{I}}+S_{I},
\end{aligned}
$$

and

$$
\begin{aligned}
\frac{d E}{d t}= & -\frac{E}{\tau_{E}}+\left(\frac{n_{D T}}{2}\right)^{2}\langle\sigma v\rangle Q_{\alpha}-P_{\text {rad }} \\
& +P_{\text {aux }}+P_{\text {ohmic }},
\end{aligned}
$$

where

$$
\begin{aligned}
n_{I}= & \text { impurity density } \\
\tau_{I}= & \text { confinement time for the impurity } \\
& \text { particles }
\end{aligned}
$$

$S_{I}$ (input) $=$ impurity injection rate.

The radiation loss $P_{\text {rad }}$ can be written as

$$
\begin{aligned}
P_{\text {rad }} & =P_{r a d}^{D T}+P_{r a d}^{\alpha}+P_{r a d}^{I} \\
& =\left[\psi_{D T}(T) n_{D T}+\psi_{\alpha}(T) n_{\alpha}+\psi_{I}(T) n_{I}\right] n_{e},
\end{aligned}
$$

where the radiation due to the DT particles is bremsstrahlung radiation $\psi_{D T}=\psi_{\text {brem }}=A_{b} \sqrt{T}$ and the radiation losses due to the alpha and impurity particles are computed according to the law

$$
\psi_{Z}(T)=\psi_{Z}^{\text {Post }}(T)=10 \sum_{i=0}^{5} A_{i}^{Z}\{\log [T(\mathrm{keV})]\},
$$

where $Z$ is the type of ion and the constant parameters $A_{i}$ can be found in Ref. 26.

The neutrality condition can be stated now as $n_{e}=$ $n_{D T}+2 n_{\alpha}+Z_{I} n_{I}$, where $Z_{I}$ is the atomic number of the impurities, whereas the total density and the energy are written as

$$
n=n_{\alpha}+n_{D T}+n_{e}+n_{I}=2 n_{D T}+3 n_{\alpha}+\left(Z_{I}+1\right) n_{I}
$$

and

$$
E=\frac{3}{2} n T \Rightarrow T=\frac{2}{3} \frac{E}{2 n_{D T}+3 n_{\alpha}+\left(Z_{I}+1\right) n_{I}} .
$$


The net plasma heating power $P$ is written now as

$$
\begin{aligned}
P= & (\text { alpha heating })-P_{\text {rad }}+P_{\text {aux }}+P_{\text {ohmic }} \\
= & \left(\frac{n_{D T}}{2}\right)^{2}\langle\sigma v\rangle Q_{\alpha}-\left[\psi_{D T} n_{D T}+\psi_{\alpha} n_{\alpha}+\psi_{I} n_{I}\right] n_{e} \\
& +P_{\text {aux }}+\eta j^{2} .
\end{aligned}
$$

The impurity line radiation is highly localized near the low temperature plasma edge, typically $\rho>0.8-0.9$ depending on $Z$ and the edge temperature. During the ITER EDA studies ${ }^{27}$ this effect was taken into account for the computation of the energy confinement time. The simple way to handle this issue is to consider only the bremsstrahlung contribution from the injected impurities when computing the net plasma heating power used in the calculation of the confinement time scaling. We use the same scaling

$$
\tau_{E}=k P_{\tau}^{-0.47}
$$

but we consider a modified net plasma heating power $P_{\tau}$ defined as

$$
\begin{aligned}
P_{\tau}= & \left(\frac{n_{D T}}{2}\right)^{2}\langle\sigma v\rangle Q_{\alpha}-\left[\psi_{D T} n_{D T}+\psi_{\alpha} n_{\alpha}+\psi_{I}^{\text {brem }} n_{I}\right] n_{e} \\
& +P_{a u x}+\eta j^{2},
\end{aligned}
$$

where $\psi_{I}^{\text {brem }}=\psi_{\text {brem }}=A_{b} \sqrt{T}$. The confinement time for the impurities are also scaled with the energy confinement time $\tau_{E}$ as

$$
\tau_{I}=k_{I} \tau_{E}
$$

\section{III.B. Control Objective}

The possible operating points of the reactor are given again by the equilibria of the dynamic equations. However, in this case we look for those operating points where $\bar{S}_{I}=0$ because we are interested in equilibrium points free of impurities. The density state variables $\bar{n}_{\alpha}, \bar{n}_{D T}$, $\bar{n}_{n}$, and $\bar{n}_{I}$, energy state variable $\bar{E}$ and inputs $\bar{S}, \bar{P}_{a u x}$ at the equilibrium, are calculated as solutions of the nonlinear algebraic equations obtained by setting the left sides in Eqs. (43) through (47) to zero when two of the plasma parameters such as $T$ and $\beta$, for example, or $\bar{P}_{\text {aux }}=0$ and $T$ or $\beta$ if we are interested in an ignition equilibrium point, are chosen arbitrarily:

$$
\begin{aligned}
& 0=-\frac{\bar{n}_{\alpha}}{\bar{\tau}_{\alpha}}+\left(\frac{\bar{n}_{D T}}{2}\right)^{2}\langle\overline{\sigma v}\rangle, \\
& 0=-\frac{\bar{n}_{D T}}{\bar{\tau}_{D T}}-2\left(\frac{\bar{n}_{D T}}{2}\right)^{2}\langle\overline{\sigma v}\rangle+\frac{\bar{n}_{n}}{\bar{\tau}_{d}}, \\
& 0=-\frac{\bar{n}_{n}}{\bar{\tau}_{d}}+\bar{S}
\end{aligned}
$$

$$
0=-\frac{\bar{n}_{I}}{\bar{\tau}_{I}},
$$

and

$$
0=-\frac{\bar{E}}{\bar{\tau}_{E}}+\bar{P},
$$

where

$$
\begin{aligned}
\bar{P}= & \bar{P}_{\tau}=\left(\frac{\bar{n}_{D T}}{2}\right)^{2}\langle\overline{\sigma v}\rangle Q_{\alpha}-\left[\bar{\psi}_{D T} \bar{n}_{D T}+\bar{\psi}_{\alpha} \bar{n}_{\alpha}\right] \bar{n}_{e} \\
& +\bar{P}_{\text {aux }}+\bar{\eta} j^{2} .
\end{aligned}
$$

Taking into account that $\bar{S}_{I}=0$ and $\bar{n}_{I}=0$ from Eq. (59), we define the deviations from the desired equilibrium values as $\tilde{n}_{\alpha}=n_{\alpha}-\bar{n}_{\alpha}, \tilde{n}_{D T}=n_{D T}-\bar{n}_{D T}, \tilde{n}_{n}=$ $n_{n}-\bar{n}_{n}, \tilde{n}_{I}=n_{I}-\bar{n}_{I}=n_{I}>0, \widetilde{E}=E-\bar{E}, \widetilde{P}_{\text {aux }}=P_{\text {aux }}-$ $\bar{P}_{\text {aux }}, \tilde{S}=S-\bar{S}$, and $\tilde{S}_{I}=S_{I}-\bar{S}_{I}=S_{I}>0$, and we write the dynamic equations for the deviations as

$$
\begin{aligned}
\frac{d \tilde{n}_{\alpha}}{d t}= & -\frac{\tilde{n}_{\alpha}}{\tau_{\alpha}}+\left(\frac{\tilde{n}_{D T}}{2}\right)^{2}\langle\sigma v\rangle+\frac{1}{2} \tilde{n}_{D T} \bar{n}_{D T}\langle\sigma v\rangle+u_{\alpha}, \\
\frac{d \tilde{n}_{D T}}{d t}= & -\frac{\tilde{n}_{D T}}{\tau_{D T}}-2\left(\frac{\tilde{n}_{D T}}{2}\right)^{2}\langle\sigma v\rangle-\tilde{n}_{D T} \bar{n}_{D T}\langle\sigma v\rangle \\
& +\frac{\tilde{n}_{n}}{\tau_{d}}+u_{D T}, \\
\frac{d \tilde{n}_{n}}{d t}= & S^{*}, \\
\frac{d \tilde{n}_{I}}{d t}= & -\frac{\tilde{n}_{I}}{\tau_{I}}+S_{I},
\end{aligned}
$$

and

$$
\frac{d \widetilde{E}}{d t}=-\frac{\widetilde{E}}{\tau_{E}}-\left[\frac{\bar{E}}{\tau_{E}}-\left[\left(\frac{n_{D T}}{2}\right)^{2}\langle\sigma v\rangle Q_{\alpha}+\eta j^{2}+u\right]\right],
$$

where

$$
\begin{aligned}
u_{\alpha} & =-\frac{\bar{n}_{\alpha}}{\tau_{\alpha}}+\left(\frac{\bar{n}_{D T}}{2}\right)^{2}\langle\sigma v\rangle \\
u_{D T} & =-\frac{\bar{n}_{D T}}{\tau_{D T}}-2\left(\frac{\bar{n}_{D T}}{2}\right)^{2}\langle\sigma v\rangle+\frac{\bar{n}_{n}}{\tau_{d}} \\
S^{*} & =-\frac{\tilde{n}_{n}}{\tau_{d}}+\tilde{S}-\frac{\bar{n}_{n}}{\tau_{d}}+\bar{S}=-\frac{n_{n}}{\tau_{d}}+S \\
u & =P_{\text {aux }}-P_{\text {rad }} .
\end{aligned}
$$


The control objective is to drive the initial perturbations in $\tilde{n}_{\alpha}, \tilde{n}_{D T}, \tilde{n}_{n}, \tilde{n}_{I}$, and $\widetilde{E}$ to zero using actuation through $P_{\text {aux }}=\bar{P}_{\text {aux }}+\widetilde{P}_{\text {aux }}, S=\bar{S}+\tilde{S}$, and $S_{I}=\tilde{S}_{I}>0$. It is important to note that in the ignition case we have $\widetilde{P}_{\text {aux }}>0$ as a constraint; we do not have anymore the possibility of modulating $P_{a u x}$ in both the positive and negative sense as we did in the subignition case. However, the additional actuator $S_{I}=\tilde{S}_{I}>0$, although constrained in sign by itself, will help us to overcome the constraint in $P_{a u x}$. Therefore, the controlled injection of impurities allows us not only to work at an ignition point but also to reject those perturbations in initial conditions that the modulation of the auxiliary power can not reject when working at a subignition point.

\section{III.C. Controller Design}

We start by looking for a control which stabilizes $\widetilde{E}$. In order to simplify the design, we choose $u$ such that

$$
\frac{\bar{E}}{\tau_{E}}-\left[\left(\frac{n_{D T}}{2}\right)^{2}\langle\sigma v\rangle Q_{\alpha}+\eta j^{2}+u\right]=0,
$$

which implies a choice of $K_{E}=1$. However, as it was discussed for the subignition case, we can choose $K_{E} \neq 1$ in order to regulate the response rate of $\widetilde{E}$. This condition means, after replacing $u$ by its expression, that we choose $P_{a u x}$ and $P_{r a d}\left(n_{I}\right)$ such that

$$
\frac{\bar{E}}{\tau_{E}}=\left(\frac{n_{D T}}{2}\right)^{2}\langle\sigma v\rangle Q_{\alpha}+\eta j^{2}-P_{r a d}+P_{a u x}=P .
$$

For the subignition case, the choice of $K_{E}=1$ helped us to avoid the computation of the solution of a nonlinear implicit equation for $P_{a u x}$. However, this is not valid anymore. Although we still know from the equilibrium Eq. (60) that $0=-\left(\bar{E} / \bar{\tau}_{E}\right)+\bar{P}, P=\bar{P}$ is no longer a solution of Eq. (71) because in the ignition case the confinement time is scaled with $P_{\tau}$ and not with $P$. Therefore, making $P=\bar{P}$ does not make $P_{\tau}=\bar{P}$. On the other hand, now it is not always possible to fulfill condition (71) modulating the auxiliary power $P_{a u x}$. In some cases we will need to find the amount of impurities that satisfies that condition and this implies the computation of the solution of a nonlinear implicit equation for $n_{I}$.

Nevertheless, although more complex, we can still find a solution for Eq. (71) and reduce Eq. (65) to

$$
\frac{d \widetilde{E}}{d t}=-\frac{\widetilde{E}}{\tau_{E}},
$$

and in this way make the subsystem $\widetilde{E}$ exponential stable since $\tau_{E}>0$. The controller that implements (71) is synthesized now in two steps:
First step: We compute $P_{a u x}$ as a solution of Eq. (71). If $P_{a u x} \geq 0$ then we keep this value for $P_{a u x}$ and let $S_{I}=0$. If $P_{\text {aux }}<0$ then we take $P_{\text {aux }}=0$ and go to the second step,

Second step: We look for the least $n_{I}=n_{I}^{*}>0$ such that

$$
\begin{aligned}
-\frac{\bar{E}}{\tau_{E}} & +\left(\frac{n_{D T}}{2}\right)^{2}\langle\sigma v\rangle Q_{\alpha}+\eta j^{2} \\
& =\left[\psi_{D T} n_{D T}+\psi_{\alpha} n_{\alpha}+\psi_{I} n_{I}\right]\left(n_{D T}+2 n_{\alpha}+Z_{I} n_{I}\right) .
\end{aligned}
$$

Defining

$$
\begin{aligned}
& \hat{n}_{I}=\tilde{n}_{I}-n_{I}^{*} \\
& f\left(\hat{n}_{I}, \widetilde{E}, \tilde{n}_{\alpha}, \tilde{n}_{D T}\right) \\
& =-\left[\frac{\bar{E}}{\tau_{E}}-\left[\left(\frac{n_{D T}}{2}\right)^{2}\langle\sigma v\rangle Q_{\alpha}+\eta j^{2}-P_{r a d}\right]\right] \\
& S_{I}=\frac{n_{I}^{*}}{\tau_{I}}+S_{I}^{*} \text {, }
\end{aligned}
$$

we can rewrite Eqs. (65) and (66) as

$$
\frac{d \hat{n}_{I}}{d t}=-\frac{\hat{n}_{I}}{\tau_{I}}+S_{I}^{*}
$$

and

$$
\begin{gathered}
\frac{d \widetilde{E}}{d t}=-\frac{\widetilde{E}}{\tau_{E}}+f\left(\hat{n}_{I}, \widetilde{E}, \tilde{n}_{\alpha}, \tilde{n}_{D T}\right), \\
f\left(0, \widetilde{E}, \tilde{n}_{\alpha}, \tilde{n}_{D T}\right)=0 .
\end{gathered}
$$

We take $V=\left(\hat{n}_{I}^{2}+\widetilde{E}^{2}\right) / 2$ as the Lyapunov function candidate, write $f=\hat{n}_{I} \phi$, where $\phi$ is a continuous function because $f\left(0, \widetilde{E}, \tilde{n}_{\alpha}, \tilde{n}_{D T}\right)=0$, and compute

$$
\dot{V}=-\frac{\hat{n}_{I}^{2}}{\tau_{I}}-\frac{\widetilde{E}^{2}}{\tau_{E}}+\hat{n}_{I}\left[S_{I}^{*}+\widetilde{E} \phi\left(\hat{n}_{I}, \widetilde{E}\right)\right]
$$

We take

$$
\begin{aligned}
& S_{I}^{*}=-\widetilde{E} \phi\left(\hat{n}_{I}, \widetilde{E}\right)-K_{I} \hat{n}_{I}, \quad K_{I} \geq 0 \\
& S_{I}=\frac{n_{I}^{*}}{\tau_{I}}-\widetilde{E} \phi\left(\hat{n}_{I}, \widetilde{E}\right)-K_{I} \hat{n}_{I},
\end{aligned}
$$

which gives $\dot{V}=-\left[\left(1 / \tau_{I}\right)+K_{I}\right] \hat{n}_{I}^{2}-\left(\widetilde{E}^{2} / \tau_{E}\right)<0$ and achieves exponential stability.

One goal of the controller synthesis process is to keep the design as simple as possible. To achieve this goal, first we define an energy confinement time of 
design $\tau_{E}^{\text {design }}=k P^{-0.47}$. Therefore, instead of solving Eq. (71) we solve

$$
\frac{\bar{E}}{\tau_{E}^{\text {design }}}=\left(\frac{n_{D T}}{2}\right)^{2}\langle\sigma v\rangle Q_{\alpha}+\eta j^{2}-P_{\text {rad }}+P_{a u x}=P .
$$

Remembering the equilibrium equation (60) for the energy, $0=-\left(\bar{E} / \bar{\tau}_{E}\right)+\bar{P}$, we can conclude that the solution for Eq. (77) is $P=\bar{P}$. Therefore, the control strategy will be to adjust $P_{\text {aux }}$ and $n_{I}$ to make $P$ constant and equal to $\bar{P}$. Results of the simulation study made a posteriori, where the controller synthesized using the approximate scaling $\tau_{E}=\tau_{E}^{\text {design }}=k P^{-0.47}$ is tested with the real system where $\tau_{E}=k P_{\tau}^{-0.47}$, show that the controller design is robust to this approximation. On the other hand, as the controller is keeping $P$ constant and equal to $\bar{P}$, there is no risk for any boost in $\tau_{E}^{\text {design }}$.

The first step of the design can be restated. We easily compute $P_{a u x}$ as

$$
P_{a u x}=\bar{P}-\left[\left(\frac{n_{D T}}{2}\right)^{2}\langle\sigma v\rangle Q_{\alpha}+\eta j^{2}-P_{\text {rad }}\right] .
$$

If $P_{a u x} \geq 0$ then we keep this value for $P_{a u x}$ and let $S_{I}=0$. If $P_{\text {aux }}<0$ then we take $P_{\text {aux }}=0$ and find the value of $n_{I}$ that solves Eq. (78) which constitutes the hard part of the second step. In order to simplify the computation of this solution we need to find an expression for the radiation losses, that can be used for the design of our controller, simpler than the one given by Post et al. in Ref. 26 and stated in Eq. (49). Stacey proposes in Ref. 28 a simpler law of the form

$$
\psi_{Z}(T)=\psi_{Z}^{\text {Stacey }}(T)=A_{b} T^{1 / 2}+A_{l} T^{-1 / 2}+A_{r} T^{-3 / 2},
$$

where $A_{b}=4.85 \times 10^{-37} \mathrm{Wm}^{3} / \sqrt{\mathrm{keV}}$ is the bremsstrahlung radiation coefficient, $A_{l}=1.8 \times 10^{-38} \mathrm{Wm}^{3} / \sqrt{\mathrm{keV}}$ is the line radiation coefficient and $A_{r}=4.1 \times 10^{-40}$ $\mathrm{Wm}^{3} / \sqrt{\mathrm{keV}}$ is the recombination radiation coefficient. Although this law is a rather rough approximation for some values of $Z$, it allows the reduction of Eq. (78), with $P_{\text {aux }}=0$, to a polynomial equation in $n_{I}$, making the search for $n_{I}^{*}$ almost trivial. With the aim of having the simplicity of (79) without losing the accuracy of (49) we search for a law of the form

$$
\psi_{Z}^{\text {aprox }}(T)=k_{b} A_{b} T^{1 / 2}+k_{l} A_{l} T^{-1 / 2}+k_{r} A_{r} T^{-3 / 2},
$$

where the constants $k_{b}, k_{l}$ and $k_{r}$ are adjusted such that $\psi_{Z}^{\text {aprox }}(T)<\psi_{Z}^{\text {Post }}(T)$ for all $T$. Figure 4 illustrates the idea for $Z_{I}=10$ (neon). The fact that $\psi_{Z}^{\text {aprox }}(T)<$ $\psi_{Z}^{\text {Post }}(T)$ guarantees that the approximate $n_{I}^{*}$ is always higher than the real $n_{I}^{*}$ and there is no risk of losing stability by not injecting enough impurities. As conclu-

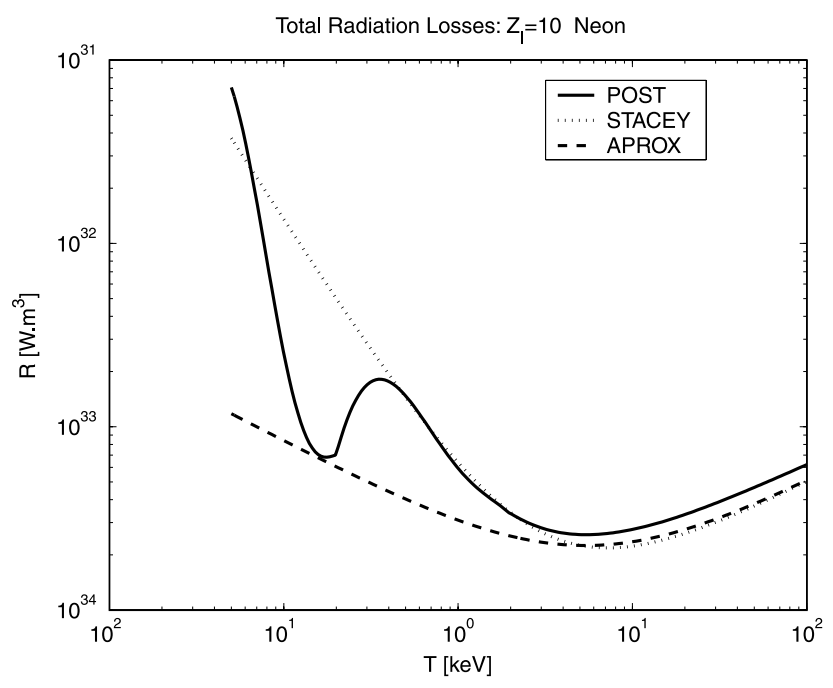

Fig. 4. Approximation of the law for radiation losses.

sion, no matter how complex the law for the radiation losses may be, there will always be a simple approximation of the radiation loss law that is good enough for control purposes.

If the reactor operates at a subignition point and the potential perturbations in initial conditions are such that they can be rejected only by the modulation of the auxiliary power $P_{a u x}$ according to the control law (78), we are in the case where impurities are not needed and $S_{I}=0$. In this case, $P$ is always equal to $\bar{P}$, Eq. (71) is always satisfied and consequently $\tau_{E}=\bar{\tau}_{E}, \tau_{\alpha}=\bar{\tau}_{\alpha}$, $\tau_{D T}=\bar{\tau}_{D T}$ and $\tau_{I}=\bar{\tau}_{I}$. If the reactor operates at an ignition point and suffers perturbations in initial conditions leading to thermal excursions, or even if it works at a subignition point but these perturbations in initial conditions are too big to be rejected only by the modulation of the auxiliary power, the injection of impurities is necessary. In this case the controller cannot ensure $P=\bar{P}$ for all time since $n_{I}$ has its own dynamics given by Eq. (65). However, it must be remarked that this transient until $P$ becomes $\bar{P}$ can be arbitrarily reduced by a proper increase of the gain $K_{I}$ if enough control energy for $S_{I}$ is available. Moreover, and more important, no matter what the length of the transient, the controller always guarantees the convergence of $n_{I}$ to $n_{I}^{*}$ and consequently the convergence of $P$ to $\bar{P}$, the satisfaction of Eq. (71) and the exponential stability of $\widetilde{E}$. The selection of the gain $K_{I}$ is always a compromise between the length of the transient and the amount of auxiliary power the reactor needs after the injection of the impurities. This selection is also a function of the atomic number $Z_{I}$, the type of impurity.

We note from Eq. (65) that $\tilde{n}_{I}$ is ISS (input-state stable) with respect to $S_{I}$. This ensures that $\tilde{n}_{I}$ will be bounded as long as $S_{I}$ is bounded, and it will be exponentially stable once $S_{I}$ becomes zero. 
After stabilizing $\widetilde{E}$ using $P_{a u x}$ and $S_{I}$ as controllers, we must focus in Eqs. (62) and (63) to achieve stability for $\tilde{n}_{D T}$ and $\tilde{n}_{\alpha}$. We apply a backstepping procedure to achieve stability of $\tilde{n}_{D T}$. Toward this goal, we start taking $\tilde{n}_{n}$ as the virtual control $w$,

$$
\begin{aligned}
\frac{d \tilde{n}_{D T}}{d t}= & -\frac{\tilde{n}_{D T}}{\tau_{D T}}-2\left(\frac{\tilde{n}_{D T}}{2}\right)^{2}\langle\sigma v\rangle-\tilde{n}_{D T} \bar{n}_{D T}\langle\sigma v\rangle \\
& +u_{D T}+\frac{w}{\tau_{d}} .
\end{aligned}
$$

Since $\left[\left(1 / \tau_{D T}\right)+\bar{n}_{D T}\langle\sigma v\rangle\right]$ is positive, we exponentially stabilize $\tilde{n}_{D T}$ taking

$$
w=\alpha\left(n_{\alpha}, n_{D T}, E\right)=\tau_{d}\left[2\left(\frac{\tilde{n}_{D T}}{2}\right)^{2}\langle\sigma v\rangle-u_{D T}\right],
$$

reducing in this way Eq. (63) to:

$$
\frac{d \tilde{n}_{D T}}{d t}=-\left[\frac{1}{\tau_{D T}}+\bar{n}_{D T}\langle\sigma v\rangle\right] \tilde{n}_{D T} .
$$

Defining now $z=\tilde{n}_{n}-\alpha \Leftrightarrow \tilde{n}_{n}=z+\alpha$, we can write

$$
\dot{z}=\dot{\tilde{n}}_{n}-\dot{\alpha}=S^{*}-\dot{\alpha} .
$$

Taking the Lyapunov function candidate $V=\left(\tilde{n}_{D T}^{2} / 2\right)+$ $\left(z^{2} / 2\right)$, from Eqs. (63) and (82) and taking into account our definition (81) for $\alpha$ we can compute

$$
\begin{aligned}
& \dot{V}= \tilde{n}_{D T} \dot{\tilde{n}}_{D T}+z \dot{z} \\
&=\tilde{n}_{D T}\left[-\left(\frac{1}{\tau_{D T}}+\bar{n}_{D T}\langle\sigma v\rangle\right) \tilde{n}_{D T}-2\left(\frac{\tilde{n}_{D T}}{2}\right)^{2}\langle\sigma v\rangle\right. \\
&\left.\quad+u_{D T}+\frac{\alpha+z}{\tau_{d}}\right]+z\left[S^{*}-\dot{\alpha}\right] \\
&=-\left[\frac{1}{\tau_{D T}}+\bar{n}_{D T}\langle\sigma v\rangle\right] \tilde{n}_{D T}^{2}+z\left[S^{*}-\dot{\alpha}+\frac{\tilde{n}_{D T}}{\tau_{d}}\right] .
\end{aligned}
$$

Taking

$$
\begin{aligned}
S^{*} & =-K_{S} z+\dot{\alpha}-\frac{\tilde{n}_{D T}}{\tau_{d}} \\
& =-K_{S}\left(\tilde{n}_{n}-\alpha\right)+\dot{\alpha}-\frac{\tilde{n}_{D T}}{\tau_{d}}
\end{aligned}
$$

and

$$
S=-K_{S}\left(\tilde{n}_{n}-\alpha\right)+\dot{\alpha}-\frac{\tilde{n}_{D T}}{\tau_{d}}+\frac{n_{n}}{\tau_{d}},
$$

with $K_{S}>0$, we have

$$
\dot{V}=-\left[\frac{1}{\tau_{D T}}+\bar{n}_{D T}\langle\sigma v\rangle\right] \tilde{n}_{D T}^{2}-K_{S} z^{2}<0,
$$

and we achieve exponential stability for the subsystem $\left(\tilde{n}_{D T}, \tilde{n}_{n}\right)$.

Replacing $u_{D T}$ by its expression given by Eq. (68), we rewrite the Eq. (81) for $\alpha$ as

$$
\begin{aligned}
\alpha\left(n_{\alpha}, n_{D T}, E\right)=\tau_{d}[ & 2\left(\frac{\tilde{n}_{D T}}{2}\right)^{2}\langle\sigma v\rangle+\frac{\bar{n}_{D T}}{\tau_{D T}} \\
& \left.+2\left(\frac{\bar{n}_{D T}}{2}\right)^{2}\langle\sigma v\rangle-\frac{\bar{n}_{n}}{\tau_{d}}\right],
\end{aligned}
$$

we can compute

$$
\begin{aligned}
\dot{\alpha}=\dot{\tau}_{d}\left[2\left(\frac{\tilde{n}_{D T}}{2}\right)^{2}\langle\sigma v\rangle+\frac{\bar{n}_{D T}}{\tau_{D T}}+2\left(\frac{\bar{n}_{D T}}{2}\right)^{2}\langle\sigma v\rangle\right] \\
+\tau_{d}\left[\langle\sigma v\rangle \tilde{n}_{D T} \dot{\tilde{n}}_{D T}+2\left(\frac{\tilde{n}_{D T}}{2}\right)^{2}\langle\dot{\sigma} v\rangle-\frac{\bar{n}_{D T}}{\tau_{D T}^{2}} \dot{\tau}_{D T}\right. \\
\left.+2\left(\frac{\bar{n}_{D T}}{2}\right)^{2}\langle\dot{\sigma} v\rangle\right]
\end{aligned}
$$

where

$$
\begin{aligned}
\langle\dot{\sigma v}\rangle & =\frac{d\langle\sigma v\rangle}{d T} \dot{T} \\
& =\frac{d\langle\sigma v\rangle}{d T}\left[\frac{\partial T}{\partial E} \dot{E}+\frac{\partial T}{\partial n_{D T}} \dot{n}_{D T}+\frac{\partial T}{\partial n_{\alpha}} \dot{n}_{\alpha}+\frac{\partial T}{\partial n_{I}} \dot{n}_{I}\right],
\end{aligned}
$$

and we compute

$$
\begin{aligned}
\frac{d\langle\sigma v\rangle}{d T}= & \langle\sigma v\rangle \\
& \times\left(\frac{-a_{1} r}{T^{r+1}}+a_{3}+2 a_{4} T+3 a_{5} T^{2}+4 a_{6} T^{3}\right)
\end{aligned}
$$


from (7) and

$$
\begin{aligned}
\frac{\partial T}{\partial E} & =\frac{2}{3} \frac{1}{2 n_{D T}+3 n_{\alpha}+\left(Z_{I}+1\right) n_{I}} \\
\frac{\partial T}{\partial n_{D T}} & =-\frac{4}{3} \frac{E}{\left(2 n_{D T}+3 n_{\alpha}+\left(Z_{I}+1\right) n_{I}\right)^{2}} \\
\frac{\partial T}{\partial n_{\alpha}} & =-2 \frac{E}{\left(2 n_{D T}+3 n_{\alpha}+\left(Z_{I}+1\right) n_{I}\right)^{2}} \\
\frac{\partial T}{\partial n_{I}} & =-\frac{2\left(Z_{I}+1\right)}{3} \frac{E}{\left(2 n_{D T}+3 n_{\alpha}+\left(Z_{I}+1\right) n_{I}\right)^{2}}
\end{aligned}
$$

from (51) and

$$
\begin{aligned}
\dot{\tau}_{i} & =k_{i} \dot{\tau}_{E} \\
& =k_{i} k(-0.47) P^{-1.47} \dot{P}
\end{aligned}
$$

from (11).

We finally compute $\dot{P}$ from (52) in terms of $\dot{n}_{\alpha}, \dot{n}_{D T}$, $\dot{n}_{I}$ and $\dot{E}$ whose values can be obtained from (62), (63), (65), and (66), respectively.

In order to finish our stability analysis we rewrite here the equation for $\tilde{n}_{\alpha}$

$$
\begin{aligned}
\frac{d \tilde{n}_{\alpha}}{d t} & =-\frac{\tilde{n}_{\alpha}}{\tau_{\alpha}}+\left(\frac{\tilde{n}_{D T}}{2}\right)^{2}\langle\sigma v\rangle+\frac{1}{2} \tilde{n}_{D T} \bar{n}_{D T}\langle\sigma v\rangle+u_{\alpha} \\
u_{\alpha} & =-\frac{\bar{n}_{\alpha}}{\tau_{\alpha}}+\left(\frac{\bar{n}_{D T}}{2}\right)^{2}\langle\sigma v\rangle .
\end{aligned}
$$

We note that $\tilde{n}_{\alpha}$ is ISS (input-state stable) with respect to $\tilde{n}_{D T}$ and $u_{\alpha}$. Therefore, since $\tilde{n}_{D T}$ is bounded (because it is exponentially stable), and $u_{\alpha}$ is bounded (because $\widetilde{E}$ is exponentially stable and $\langle\sigma v\rangle$ is a bounded function), $\tilde{n}_{\alpha}$ will be bounded for all time. In addition, once $E$ converges to $\bar{E}(\widetilde{E} \rightarrow 0), n_{D T}$ converges to $\bar{n}_{D T}\left(\tilde{n}_{D T} \rightarrow 0\right)$, and $n_{I}$ converges to $\bar{n}_{I}=0$ this equation reduces to

$$
\begin{aligned}
\frac{d \tilde{n}_{\alpha}}{d t} & =-\frac{\tilde{n}_{\alpha}}{\bar{\tau}_{\alpha}}+u_{\alpha}^{*}, \\
u_{\alpha}^{*} & =-\frac{\bar{n}_{\alpha}}{\bar{\tau}_{\alpha}}+\left(\frac{\bar{n}_{D T}}{2}\right)^{2}\langle\sigma v\rangle .
\end{aligned}
$$

Repeating the arguments presented for the subignition case we note that $u_{\alpha}^{*}$ has the same sign as $-\left(\tilde{n}_{\alpha} / \bar{\tau}_{\alpha}\right)$ and vanishes when $\tilde{n}_{\alpha}$ vanishes $(\langle\sigma v\rangle=\langle\overline{\sigma v}\rangle)$. This allows us to conclude exponential stability for $\tilde{n}_{\alpha}$.

\section{III.D. Simulation Results}

In this section the performance of the controller stabilizing the equilibrium point characterized by those values given in Table III is studied through computer simulations. For all the simulations presented here, the
TABLE III

ITER Equilibrium Point 2-Ignition Point

\begin{tabular}{|lll|}
\hline $\bar{T}$ & Temperature & $7.5 \mathrm{keV}$ \\
$\bar{n}_{e}$ & Electron density & $1.20 \times 10^{20} \mathrm{~m}^{-3}$ \\
$\bar{f}_{\alpha}$ & Alpha fraction & $5.53 \%$ \\
$\bar{\beta}$ & Beta & $3 \%$ \\
$\bar{n}_{\alpha}$ & Alpha density & $6.64 \times 10^{18} \mathrm{~m}^{-3}$ \\
$\bar{n}_{D T}$ & DT density & $1.07 \times 10^{20} \mathrm{~m}^{-3}$ \\
$\bar{E}$ & Energy & $4.21 \times 10^{5} \mathrm{~J} \cdot \mathrm{m}^{-3}$ \\
$\bar{P}_{\text {aux }}$ & Auxiliary power & $0 \mathrm{~W} \cdot \mathrm{m}^{-3}$ \\
$\bar{S}$ & Fuel rate & $5.52 \times 10^{18} \mathrm{~m}^{-3} \cdot \mathrm{s}^{-1}$ \\
\hline
\end{tabular}

controller gains $K_{S}=0.1$ and $K_{E}=1$, a maximum auxiliary power ramp rate $R_{\text {aux }}=20 \mathrm{MW} / \mathrm{s}$ and a scale factor $f=f^{H}=0.85$ for the energy confinement time (11) have been used. When $P<P_{t h r}$ we adopt $f=f^{L}=f^{H} / 2$. It should be noted that our controller can be independent of $k_{I}$ choosing a sufficiently high value for $K_{I}$. Consequently it tolerates any size of uncertainty in this parameter. Therefore the choice of $k_{I}=10$ can be considered completely arbitrary and with the only purpose of the simulation.

Since we have introduced in our model the losses due to line and recombination radiations, in addition to bremsstrahlung radiation, we are not restricted to the use of low $Z$ impurities. It can be noted that for low $Z$ impurities, the losses are mainly due to the bremsstrahlung radiation for a fusion reactor temperature. However, as $Z$ increases the contributions of the line and recombination radiations become more and more important.

The controller designed shows capability of rejecting different types of large perturbations in initial conditions. Figure 5 shows a tested domain of stability for

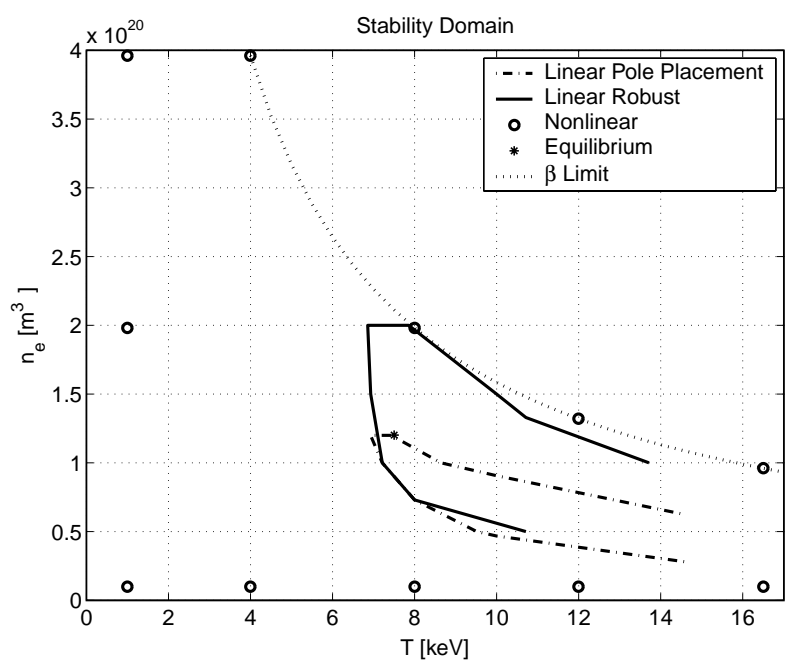

Fig. 5. Stability domain comparison. 
the nonlinear controller. This study is carried out generating initial perturbations around the equilibrium 2 for $T$ and $n_{e}$ and keeping the alpha-particle fraction $f_{\alpha}:=n_{\alpha} / n_{e}$ equal to that of the equilibrium. The figure compares its performance with other two controllers synthesized by linear pole placement ${ }^{12}$ and linear robust ${ }^{13}$ techniques, for a linearization point very close to the equilibrium point 2 , which use mainly the same dynamical model presented here but considering only the fueling rate as actuator. While the boundaries shown for the linear controllers are absolute, for the nonlinear controller they only indicate the limits within which we performed our tests. Tests exceeding the Troyon $\beta$ limit are not shown. However if the MHD stability conditions were not violated, the controller would reject also initial perturbations in this area. This is also the case for the density limit. Although the density limit is not shown in the figure, it can be appreciated that some of the perturbations in initial conditions that are rejected by the controller may exceed this limit. On the other hand, some of these perturbations in initial conditions may also exceed those stability boundaries that depend on profile effects. The goal of this controller is the stabilization of the burn condition, not the control of disruptions, and we propose arbitrary perturbations in the initial conditions to show through simulations that the controller is achieving its goal. We are aware that the proposed perturbations in the initial conditions for simulation purposes can lead to disruptions for which the controller is not prepared to deal with.

The robustness of our controller was also studied against those of the linear controllers. Figure 6a shows the regions of stability against uncertainty in the parameter $k_{\alpha}$ whose nominal value is equal to 7 when the system suffers perturbations in the initial temperature. Again, the region shown for the nonlinear controller is not a limit. With the sole objective to show its performance we tested it against uncertainties up to $400 \%$ and perturbations for initial $T$ between $-90 \%$ and $100 \%$.

The robustness of the controller against uncertainties of the parameter $k_{d}$ also has to be studied. Figure $6 \mathrm{~b}$ shows the regions of stability against uncertainty in the parameter $k_{d}$ whose nominal value is equal to 1 when the system suffers perturbations in the initial temperature. Again, the region shown for the nonlinear controller is not a limit. With the sole objective to show its performance we tested it against uncertainties up to $400 \%$ and perturbations for initial $T$ between $-90 \%$ and $100 \%$. The figure compares the robustness of the nonlinear controller with other controller synthesized by linear robust techniques ${ }^{14}$ for a linearization point very close to the equilibrium point considered here. However, we have to mention a difference between both controllers; while the nonlinear controller was synthesized here for a nominal value of 1 , the robust linear controller was synthesized for the nominal no-lag case.

Figure 7 shows the response of the system against a $50 \%$ perturbation in the temperature initial condition. We can note how the introduction of the controlled impurity injection as actuator allows us to deal successfully with a much larger set of positive perturbations in $T$. The constraint given by $R_{\text {aux }}$ does not represent any risk of loss of stability. However, as $R_{\text {aux }}$ reaches its lower value of $10 \mathrm{MW} / \mathrm{s}$ the system gets closer to show a transition to the $\mathrm{L}$ mode for recovering later. This happens after the injection of impurities because $P_{\text {aux }}$ is not fast enough to keep $P=\bar{P}$. From the figure showing the injection of impurities during the first seconds of the simulation, we can note that $S_{I}$ is turned off before $n_{I}$ reaches $n_{I}^{*}$. This is the only effect of calculating $n_{I}^{*}$ using $\psi_{Z}^{a p r o x}$ since the $n_{I}^{*}$ computed is higher than the real one.
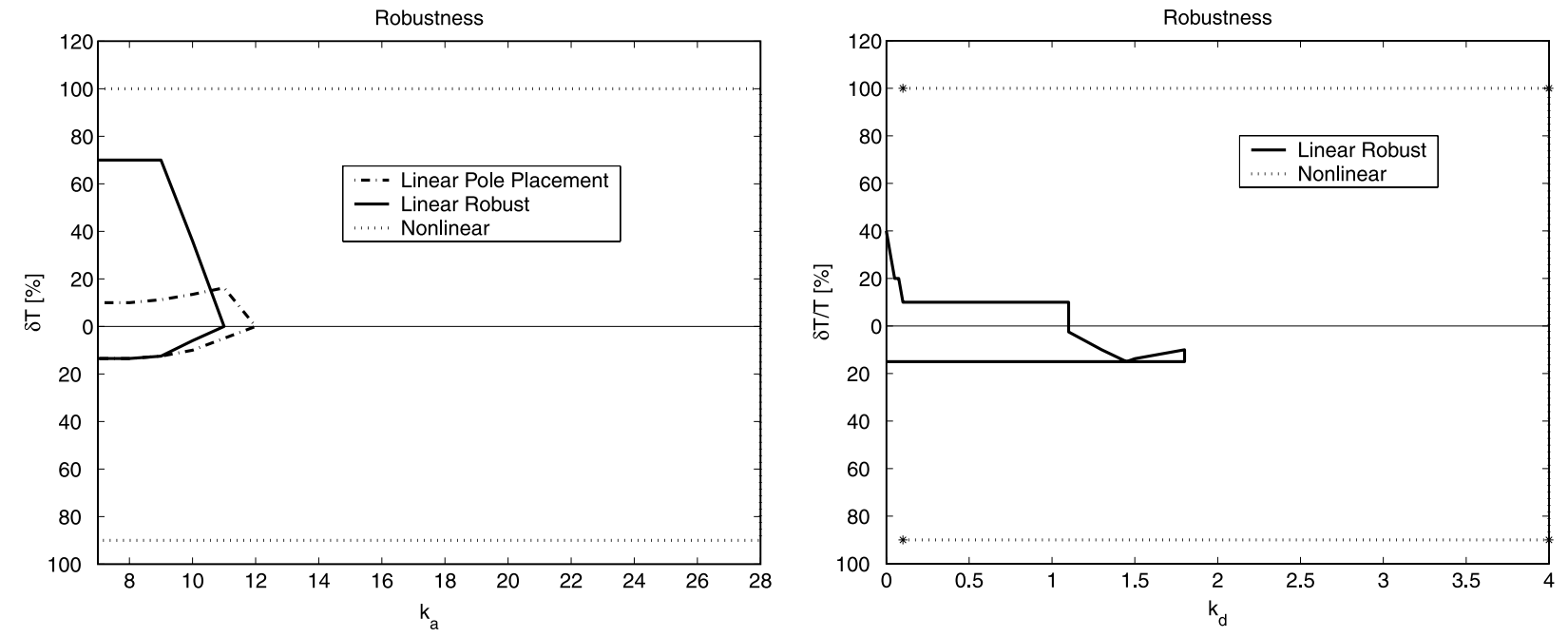

Fig. 6. Robustness against uncertainty in $k_{a}$ and $k_{d}$. 

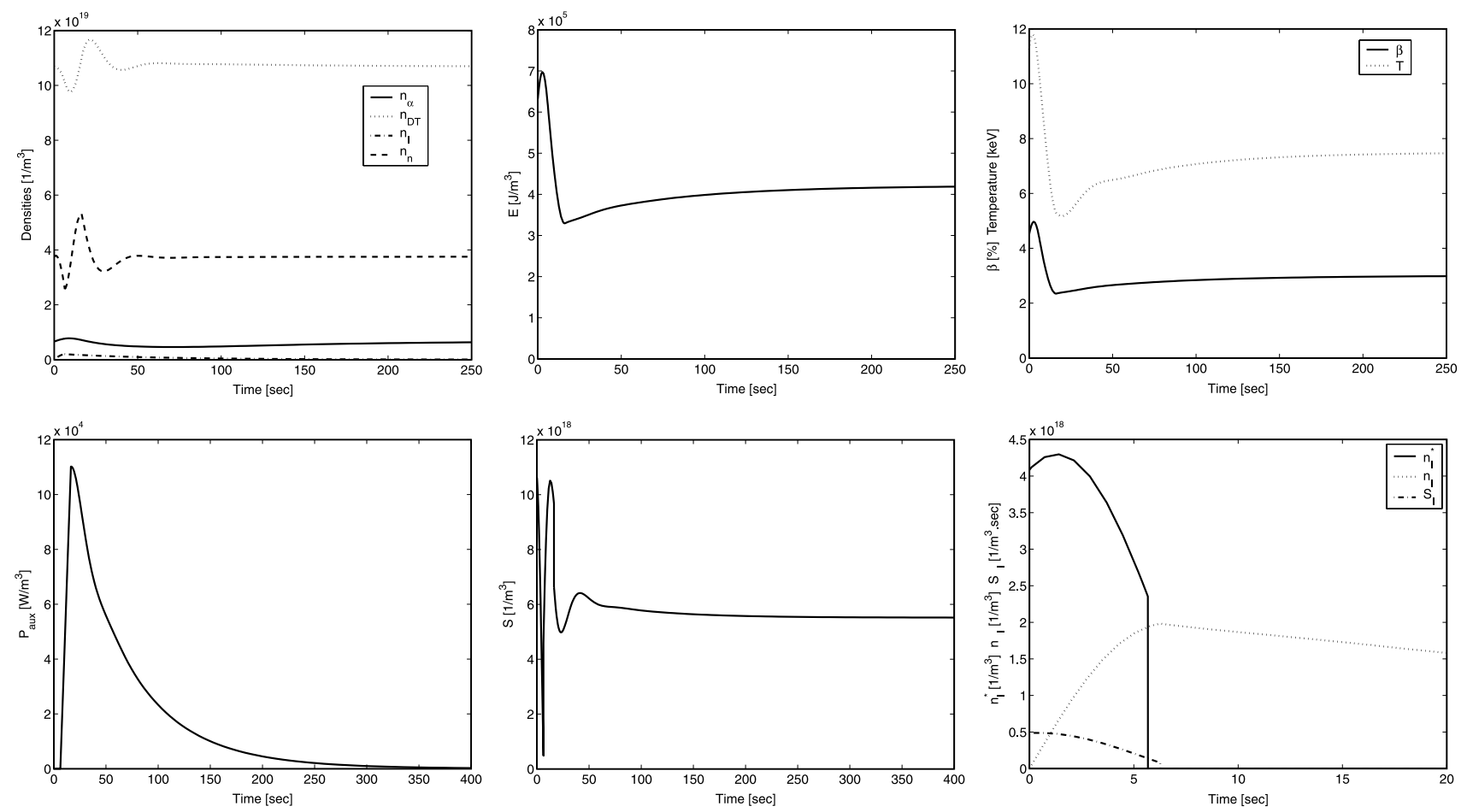

Fig. 7. With control, even under initial perturbation of $50 \%$ in $T$, the system returns to the desired equilibrium $\left(Z_{I}=10, K_{I}=0.1\right.$, $\left.K_{S}=0.1, K_{E}=1\right)$.

\section{CONCLUSIONS AND FUTURE WORK}

This new approach to the problem of burn control allows us to deal with perturbations in initial conditions that were unmanageable until now. The information taken into account by the controller when it is synthesized using the full nonlinear model makes it capable of dealing with a larger set of perturbations in initial conditions. On the other hand, the multi-input nature of the controller allows it to reject large perturbations in initial conditions leading to both thermal excursion and quenching. In addition, the effectiveness of the controller does not depend on whether the operating point is an ignition or a subignition point.

Since the nonlinear controller depends parametrically on the equilibrium point, it can drive the system from one equilibrium point to another allowing in this way the change of power, other plasma parameters, and ignition conditions. No scheduled controllers are necessary and the same control law is valid for every equilibrium point.

A Lyapunov based controller guarantees us that the state trajectory of the system does not escape outside the initial domain of attraction. This fact is very important when we try to avoid disruptive instabilities in the plasma. If the domain of attraction defined by a level surface of the Lyapunov function that contains the initial perturbation does not contain any disruptive instability point, the controller guarantees us that no possible disruptive instability point can be reached during the evolution of the system towards its equilibrium.

It must be noted that this approach can be extended to the use of any other energy confinement time scaling (11) based on the net heating power. Therefore, the controller designed can be used in other fusion reactors whose energy confinement time scaling is proportional to some power of the net heating power and is not restricted to the ITER scaling used in this work. On the other hand, this approach can also be easily extended to newer ITER scalings. We also point out that in this work we have used empirically derived expressions for the energy confinement time. The experiments used to obtain this empirical expression are usually performed under timestationary states. By definition, during a transient state, such as occurs during a thermal excursion, the plasma condition is evolving and thus it is not clear whether the empirical energy confinement time can be safely applied. It is likely that for larger excursions away from equilibrium (i.e., when $\left.(1 / \widetilde{E})(d \widetilde{E} / d t)>1 / \tau_{E}\right)$ the empirical expression for $\tau_{E}$ may break down. In this case one would need to find an alternate expression for energy confinement, or perhaps utilize a one-dimensional model to incorporate the evolution of plasma profiles that is likely to occur during larger transients.

Through the use of nonlinear backstepping it was possible to synthesize a controller which is independent 
of the reactor fueling system allowing either pellet injection or gas puffing. The controller can deal with arbitrary values of $k_{d}$. It must be mentioned that the control law (83) for $S$ is remarkably simplified when the controller does not need to inject impurities in the reactor. In this case the control law (78) for $P_{a u x}$ ensures $P=\bar{P}$ and consequently $\tau_{d}=\bar{\tau}_{d}$. Therefore, the expression for $\dot{\alpha}$ in (84) adopts a much simpler form. In addition, the combination of a singular perturbation and a passivity approach allows us to regulate, through the gain $K_{I}$, the compromise between the actuation force in control law (76) for $S_{I}$ and the energy excursion.

Simulation results show good robustness properties against uncertainties in the confinement times. The control laws (78), (76), and (83) are functions of $k_{D T}$ and $k_{I}$. However the dependence on $k_{I}$ in (76) can be eliminated choosing $K_{I}$ big enough and the dependence on $k_{D T}$ in (83) can be avoided with a slight modification in the design that is not presented in this work. The boundedness of the system solutions is achieved for any kind and size of perturbation in initial conditions regardless of the size and nature of the uncertainty. The controller is always robust against uncertainties in $k_{I}$ (when $K_{I}$ is big enough), is always able to drive $E \rightarrow \bar{E}$ regardless of the uncertainty type and, in addition, is able to drive $n_{D T} \rightarrow$ $\bar{n}_{D T}$ when there is no uncertainty in $k_{D T}$. In order to drive the system to the equilibrium point corresponding to the actual values of the confinement times, and to avoid spending control effort on handling the uncertainties in an unstructured (non-parametric) manner, a nonlinear adaptive control law should be synthesized.

Besides the uncertainties in the confinement time constants, the model can also present structural uncertainties. In addition to the variation of the energy confinement time during the transients already mentioned above, we should add the variation of some parameters like the reactivity rate with the kinetic profiles. In order to apply the proposed control scheme in a real plasma the sensitivity of the stability on the model and measurement uncertainties must be studied. There are systematic control techniques to deal with these uncertainties that can complement the present design. As a major research topic in its own right, this issue does not lie within the scope of this paper and will be part of future work.

One possible extension of this work involves developing a one-dimensional dynamic model. In this way we would not only achieve results for a plant that is closer to reality but also gain expertise that could be directly applicable to other problems in control of nuclear fusion. Problems like the transport control, the improvement of the energy confinement time and MHD stability, among others, require control of not only the values of the density, temperature, and current, but also of their profiles. In order to tackle these problems, a nonlinear controller should be synthesized for a model incorporating spatial information.

\section{APPENDIX}

\section{LYAPUNOV STABILITY BASICS}

For the benefit of the reader, in this Appendix we review the basics of Lyapunov stability theory which is extensively used in this paper. Consider the system

$$
\dot{x}=f(x, u),
$$

where $x$ (state variable) and $u$ (control input) are vectorvalued functions of time. We are interested in finding a feedback control law

$$
u=k(x),
$$

to achieve some desired property, for example, stability. A point $x=x_{e}$ such that

$$
f\left(x_{e}, k\left(x_{e}\right)\right)=0
$$

is called an equilibrium.

An equilibrium $x=x_{e}$ of (A.1) and (A.2) is globally asymptotically stable if there exists a continuously differentiable function $V(x)$ such that

$$
\begin{gathered}
V(x)>0 \text { for all } x \neq 0 \text { and } V(0)=0 \\
V(x) \rightarrow \infty \text { as }|x| \rightarrow \infty \\
\dot{V}=\frac{d V}{d x} f(x, k(x))<0 \text { for all } x \neq 0 .
\end{gathered}
$$

For example, if we can find a quadratic $V=x^{T} P x$ with $\dot{V}=-x^{T} Q x, P, Q>0$, all the stability conditions are satisfied.

The problem of finding a Lyapunov function $V(x)$, even for a system we already know is stable, is very difficult in general. It is even more difficult when we have to find $V(x)$ and the feedback law $k(x)$ simultaneously.

While for linear systems other (eigenvalue-type) stability tests exist, like, for example, Routh-Hurwitz, for nonlinear systems some form of Lyapunov analysis is the only tool available. See Ref. 25 for a complete approach to the Lyapunov stability theory.

\section{REFERENCES}

1. G. T. SAGER, “Review: Tokamak Burn Control,” DOE/ ER/52127-36, Fusion Studies Laboratory, University of Illinois (1988).

2. S. MIRNOV et al., "Chapter 8: Plasma Operation and Control," Nucl. Fusion, 39, 12, 2251, International Atomic Energy Agency, Vienna, Austria (Dec. 1999).

3. J. E. VITELA and J. J. MARTINELL, "Burn Conditions Stabilization with Artificial Neural Networks of Subignited Thermonuclear Reactors with Scaling Law Uncertainties," Plasma Phys. Control. Fusion, 43, 99 (2001). 
4. J. E. VITELA and J. J. MARTINELL, "Stabilization of Burn Conditions in a Thermonuclear Reactor Using Artificial Neural Networks," Plasma Phys. Control. Fusion, 40, 295 (1998).

5. J. MANDREKAS and W. M. STACEY, Jr., "Evaluation of Different Control Methods for the Thermal Stability of the International Thermonuclear Experimental Reactor," Fusion Technol., 19, 57 (1991).

6. S. W. HANEY, L. J. PERKINS, J. MANDREKAS, and W. M. STACEY, Jr., "Active Control of Burn Conditions for the International Thermonuclear Experimental Reactor," $\mathrm{Fu}$ sion Technol., 18, 606 (1990).

7. D. ANDERSON, T. ELEVANT, H. HAMEN, M. LISAK, and H. PERSSON, "Studies of Fusion Burn Control," Fusion Technol., 23, 5 (1993).

8. L. BROMBERG, J. L. FISHER, and D. R. COHN, "Active Burn Control of Nearly Ignited Plasmas," Nucl. Fusion, 20, 2 (1980).

9. E. A. CHANIOTAKIS, J. P. FREIDBERG, and D. R. COHN, "CIT Burn Control Using Auxiliary Power Modulation," Proc. 13th IEEE/NPSS Symp. Fusion Engineering, Vol. 1, p. 400, Institute of Electrical and Electronics Engineers/ Nuclear and Plasma Sciences Society (1990).

10. S. W. HANEY and L. J. PERKINS, "Operating Point Selection and Burn Stability Control for the International Thermonuclear Experimental Reactor," Proc. 13th IEEE/NPSS Symp. Fusion Engineering, Vol. 1, p. 396, Institute of Electrical and Electronics Engineers/Nuclear and Plasma Sciences Society (1990).

11. D. E. T. F. ASHBY and M. H. HUGHES, "Dynamic Burn Control of a Tokamak Reactor by Fuel Injection," Nucl. Fusion, 20, 4, 451 (1980).

12. W. HUI and G. H. MILEY, "Burn Control by Refueling," Bull. Am. Phys. Soc., 37, 6, 1399 (1992).

13. B. A. BAMIEH, W. HUI, and G. H. MILEY, "Robust Burn Control of a Fusion Reactor by Modulation of the Refueling Rate," Fusion Technol., 25, 3, 318 (1994).

14. W. HUI, K. FISCHBACH, B. BAMIEH, and G. H. MILEY, "Effectiveness and Constraints of Using the Refueling System to Control Fusion Reactor Burn," Proc. 15th IEEE/ NPSS Symp. Fusion Engineering, Vol. 2, p. 562, Institute of Electrical and Electronics Engineers/Nuclear and Plasma Sciences Society (1994).

15. D. A. PLUMMER, "Fusion Reactor Control," Proc. 16th IEEE/NPSS Symp. Fusion Engineering, Vol. 2, p. 1186, Institute of Electrical and Electronics Engineers/Nuclear and Plasma Sciences Society (1995).
16. A. SESTERO, "Proposed Scenario for Burn Control in Tokamak Reactors," Nucl. Technology/Fusion, 4, 437 (1983).

17. O. MITARAI and K. MURAOKA, "A Proposed Set of Diagnostics for Core Ignition Burn Control in a Tokamak Reactor," Nucl. Fusion, 39, 6, 725 (1999).

18. G. SAGER, G. H. MILEY, and I. MAYA, "Optimal Control Theory Applied to Fusion Plasma Thermal Stabilization," Fusion Technol., 8, 1795 (1985).

19. M. A. FIRESTONE and C. E. KESSEL, "Plasma Kinetics Control in a Tokamak," IEEE Trans. Plasma Sci., 19, 1, 29 (1991).

20. G. H. MILEY and V. VARADARAJAN, "On Self-Tuning Control of Tokamak Thermokinetics," Fusion Technol., 22, 425 (1992).

21. V. FUCHS, M. M. SHOUCRI, G. THIBAUDEAU, L. HARTEN, and A. BERS, "High-Q Thermally Stable Operation of a Tokamak Reactor," IEEE Trans. Plasma Sci., PS-11, 1, 4 (Mar. 1983).

22. L. M. HIVELY, "Convenient Computational Forms for Maxwellian Reactivities," Nucl. Fusion, 17, 4, 873 (1977).

23. N. A. UCKAN, "Confinement Capability of ITER-EDA Design," Proc. 15th IEEE/NPSS Symp. Fusion Engineering, Vol. 1, p. 183, Institute of Electrical and Electronic Engineers/ Nuclear and Plasma Sciences Society (1994).

24. Y. SHIMOMURA et al., "ITER-FEAT Operation," Nucl. Fusion, 41, 3, 309 (2001).

25. H. K. KHALIL, Nonlinear Systems, 2nd ed., Prentice Hall, Englewood Cliffs, New Jersey (1996).

26. D. E. POST, R. V. JENSEN, C. B. TARTER, W. H. GRASBERGER, and W. A. LOKKE, "Steady-State Radiative Cooling Rates for Low-Density, High-Temperature Plasmas," At. Data Nucl. Data Tables, 20, 5 (Nov. 1977).

27. N. A. UCKAN (compiled by), "ITER Physics Design Guidelines: 1989," ITER Documentation Series No. 10, International Atomic Energy Agency (1990).

28. W. STACEY, Fusion: An Introduction to the Physics and Technology of Magnetic Confinement Fusion, John Wiley \& Sons, New York (1984).

29. N. A. UCKAN, J. HOGAN, W. HOULBERG, J. GALAMBOS, L. J. PERKINS, S. HANEY, D. POST, and S. KAYE, "ITER Design: Physics Basis for Size, Confinement Capability Power Levels and Burn Control," Fusion Technol., 26, 327 (1994).

FUSION SCIENCE AND TECHNOLOGY VOL. 43 JAN. 2003 
Eugenio Schuster (BE, electrical engineering, University of Buenos Aires, Argentina, 1993; BE, nuclear engineering, Balseiro Institute, Argentina, 1998; MS, aerospace engineering, University of California, San Diego, 2000) is currently working on his $\mathrm{PhD}$ thesis in the Department of Mechanical and Aerospace Engineering at University of California, San Diego. He is interested in the application of nonlinear control techniques to the problems of magnetic and kinetic control, MHD stability, transport reduction, and MHD flow control in fusion reactors.

Miroslav Krstić ( $\mathrm{PhD}$, electrical engineering, University of California, Santa Barbara, 1994) is professor and vice chair in the Department of Mechanical and Aerospace Engineering at University of California, San Diego. He is well known for his research on nonlinear adaptive control. His research interests include nonlinear, adaptive, robust, and stochastic control theory for finite dimensional and distributed parameter systems, and applications to propulsion systems, flows, and fusion reactor instabilities.

George Tynan ( $\mathrm{PhD}$, engineering science, University of California, Los Angeles, 1991) is associate professor in the Department of Mechanical and Aerospace Engineering at University of California, San Diego. He is also affiliated with the PISCES Plasma-Surface Interaction Experimental Group and with UCSD Fusion Energy Research Program. His research interests include plasma processing of materials, controlled shear decorrelation experiments, plasmamaterial interactions for nuclear fusion, nanocluster-plasma interactions for astrophysical and technological applications, and turbulent transport in plasmas. 